\title{
GEOMETRIC SYZYGIES OF ELLIPTIC NORMAL CURVES AND THEIR SECANT VARIETIES
}

\author{
HANS-CHRISTIAN GRAF V. BOTHMER AND KLAUS HULEK
}

\begin{abstract}
We show that the linear syzygy spaces of elliptic normal curves, their secant varieties and of bielliptic canonical curves are spanned by geometric syzygies.
\end{abstract}

\section{INTRODUCTION}

Minimal free resolutions of projective varieties $X \subset \mathbb{P}^{n}$ have received considerable attention in the last years. The aim is to understand the connection between the geometry of $X$ and the minimal free resolution of its ideal $I_{X}$. In 1984 Green and Lazarsfeld ML84 found a way to construct some so called geometric syzygies of $I_{X}$ from certain linebundles $\mathcal{L}$ on $X$. For canonical curves Green has conjectured, that these geometric syzygies determine the shape of the minimal free resolution.

Conjecture 1.1 (Green). Let $C \subset \mathbb{P}^{g-1}$ be a canonical curve without geometric pth linear syzygies. Then $C$ as no p-th linear syzygies at all.

This was recently proved by Voisin Voi02, Voi03 for general $k$-gonal curves of genus $g$ with $k \geq g / 3$. and before that by Teixidor TiB02 for general $k$-gonal curves with $k \leq(g+7) / 3$. We are, therefore, now at a point where it makes sense to ask more detailed questions about the connection between geometric syzygies and the minimal free resolution. A simple dimension count from Brill-Noether theory shows that one cannot expect all syzygies to be geometric. However, one can ask whether the geometric syzygies span, i.e.

Question 1.2. Do the geometric p-th syzygies span the spaces of all $p$-th syzygies of a given variety $X \subset \mathbb{P}^{n}$ ?

The answer to this question is known to be positive in some cases. For the 0-th syzygies (quadrics) of canonical curves this is a theorem of Green Gre84b. It is also true for the 1-st syzygies of general curves of genus $g \geq 9$ by vB00 and for 2-nd syzygies of general canonical curves of genus 8 by vB02. Furthermore Eusen Eus94 proves this for all syzygies of trigonal canonical curves. Since one can easily check it for canonical curves which are isomorphic to a plane quintic [ vB00] the case of Clifford index 1 is, therefore, solved.

Going in a different direction the answer is also positive for rational normal curves and rational normal scrolls (almost by definition). On the other hand 
it is not true for K3-surfaces $S \subset \mathbb{P}^{g}$ with Picard number 1 since these do not lie on rank 4 quadrics.

In this paper we give new results in three different directions. First we give a positive answer to the above question for all syzygies of elliptic normal curves. Secondly we prove at the same time an analogous result for the higher secant varieties of elliptic normal curves, providing further examples of higher dimensional varieties that do have syzygy spaces generated by geometric syzygies. Thirdly, we use our results to solve the question for bielliptic canonical curves of Clifford index 2 .

It is also interesting to look at the variety of geometric $p$-th syzygies inside the projectivised space of all $p$-th syzygies. We show that for elliptic normal curves and their secant varieties these varieties of geometric syzygies contain nondegenerate elliptic scrolls. One might hope that these are projectively normal, but we show that this hardly ever the case.

Our paper is organised as follows. In sections 2,3 and 4 we recall the definition and basic properties of graded Betti numbers, linear strands and geometric syzygies. In sections [5] [6] and 7 we consider families $X$ over a base $B$ whose fibres $X_{b}$ have minimal degree linear strands with identical Betti numbers. We introduce the notion of a family of linear strands in this situation and consider the union $\cup X=\cup X_{b}$ and the intersection $\cap X=\cap X_{b}$ of fibres. Their linear strands are closely related to the cohomology of the family of linear strands. These two constructions are crucial ingredients in our proofs. In section 8 we turn to elliptic normal curves and their higher secant varieties. We investigate the geometry of these secant varieties and compute their minimal free resolution. In Section 9 we use this information to construct a family of linear strands whose union of fibres is the $d$-th secant variety $\operatorname{Sec}_{d}=\operatorname{Sec}_{d} E$ of an elliptic curve $E$ and whose intersection of fibres is $\operatorname{Sec}_{d-1}=\operatorname{Sec}_{d-1} E$. This and the symmetry of the minimal free resolution of $\operatorname{Sec}_{d-1}$ allows us to prove the geometric syzygy conjecture for these secant varieties in Section 10. We also note there, that the varieties of geometric syzygies considered in the proof are almost never projetively normal. This failure of projective normality is explained in Section [11. It turns out that the missing sections of the geometric syzygy varieties of $\mathrm{Sec}_{d-1}$ make up the minimal free resolution of $\operatorname{Sec}_{d}$. Finally in Section Section 12 we prove the geometric syzygy conjecture for bielliptic canonical curves.

\section{Notation}

Throughout this paper $E \subset \mathbb{P}^{n-1}$ will be an elliptic normal curve of degree $n$. We denote the origin of $E$ by $A$ and without loss of generality we can assume that $\mathcal{O}_{E}(1) \cong \mathcal{O}_{E}(n A)$. The Jacobian of $E$ of degree $d$ complete linear series will be denoted by $\mathrm{Jac}_{d}:=\mathrm{Jac}_{d} E$. We will also consider the $d$-secant variety $\operatorname{Sec}_{d}:=\operatorname{Sec}_{d} E$ of $E$ in $\mathbb{P}^{n-1}$.

More generally, if $X \subset \mathbb{P}^{n-1}$ is any projective variety, we denote its minimal free resolution by

$$
F_{X}^{\bullet} \rightarrow I_{X} \quad \text { or } \quad F_{X}^{\bullet}[1] \rightarrow \mathcal{O} \rightarrow \mathcal{O}_{X}
$$


where we consider $F_{X}^{\bullet}$ as a bounded cochain complex

$$
F_{X}^{\bullet}: 0 \rightarrow \cdots \rightarrow F_{X}^{-2} \rightarrow F_{X}^{-1} \rightarrow F_{X}^{0}
$$

with cohomology concentrated in degree 0 .

For any free cochain complex $F^{\bullet}$ we write $F^{i}:=\oplus_{j} F_{j}^{i} \otimes \mathcal{O}(i-j)$ where the $F_{j}^{i}$ vector spaces. Here and in the rest of the paper $\mathcal{O}$ will mean $\mathcal{O}_{\mathbb{P}^{n-1}}$, similarly we set $\Omega^{p}:=\Omega_{\mathbb{P}^{n-1}}^{p}$.

The dimensions

$$
\beta_{i j}=\operatorname{dim} F_{j}^{i}
$$

are called graded Betti numbers of $X$.

Sometimes we will write more shortly

$$
F^{i}=\oplus_{j} \mathcal{O}(i-j)^{\beta_{i j}}
$$

or collect the graded Betti numbers $\beta_{i j}$ in a so-called Betti diagram:

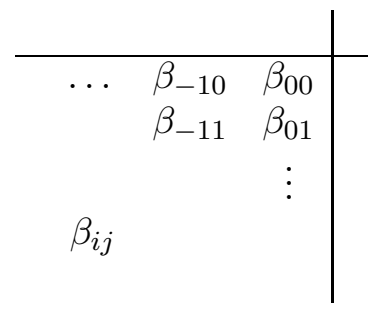

For better readability we will write a dash ("-") if $\beta_{i j}=0$.

Example 2.1. The rational normal curve $X \subset \mathbb{P}^{3}$ of degree 3 has minimal free resolution

$$
0 \rightarrow \mathcal{O}(-3)^{2} \rightarrow \mathcal{O}(-2)^{3} \rightarrow \mathcal{O} \rightarrow \mathcal{O}_{X} \rightarrow 0 .
$$

The corresponding Betti diagram is therefore

$$
\begin{array}{ccc}
- & - & 1 \\
2 & 3 & -
\end{array}
$$

Notice that this notation is dual to the one used by the computer program Macaulay GS. To obtain the diagrams calculated by this program one has to take the reflection of our diagrams with respect to a vertical line.

\section{LiNEAR StRANDS}

To study the minimal free resolution $F_{X}^{\bullet} \rightarrow I_{X}$ of a variety $X$ it is often useful to linearise its information. We will look at subcomplexes of $F_{X}^{\bullet}$ whose differentials are given by matrices of linear forms.

Definition 3.1. Let $I_{X}$ be an ideal sheaf on $\mathbb{P}^{n-1}$, and

$$
F_{X}^{\bullet} \rightarrow I_{X}
$$

a minimal free resolution of $I_{X}$. We define the complex $F_{X}^{\bullet}(d)$ by

$$
F_{X}^{i}(d)=F_{d}^{i} \otimes \mathcal{O}(i)=\mathcal{O}(i)^{\beta_{i+d, d}}
$$

with the differentials induced from $F_{X}^{\bullet}$. We call

$$
F_{X}^{\bullet}(d)[-d] \rightarrow I_{X}
$$


the degree $d$ linear strand of $I_{X}$, since the differentials of $F_{X}^{\bullet}(d)$ are given by matrices of linear forms, and the map to $I_{X}$ is defined by polynomials of degree $d$.

Definition 3.2. Let $s:=s(X)$ be the smallest integer such that $H^{0}\left(I_{X}(s)\right) \neq 0$. Then $s$ is called the postulation of $X . F_{X}^{\bullet}(s)$ is called the minimal degree linear strand of $X$.

Example 3.3. Let $F_{X}^{\bullet} \rightarrow I_{X}$ be the minimal free resolution of the rational normal curve in $\mathbb{P}^{3}$. Since $I_{X}$ is generated by quadrics, its minimal degree linear strand is $F_{X}^{\bullet}(2)$. Its Betti diagram is

$$
23--
$$

Remark 3.4. $F_{X}^{\bullet}(d)[-d]$ is a subcomplex of $F_{X}^{\bullet}$.

Remark 3.5. Notice that by Hilbert's syzygy theorem, the linear strand $F_{X}^{\bullet}(d)[-d]$ is zero in degrees smaller than $-n$.

We can calculate the linear strand of a scheme $X \subset \mathbb{P}^{n-1}$ by Koszul cohomology:

Lemma 3.6. Let $d \leq s(X)$. Then we have

$$
F_{d}^{i}=H^{0}\left(I_{X} \otimes \Omega^{-i-d}(-i)\right)
$$

for the degree $d$ linear strand of $X$.

Proof. By Koszul cohomology Gre84a] the vector space $F_{d}^{i}$ is the middle cohomology of

$$
\Lambda^{i-d+1} V \otimes\left(I_{X}\right)_{d-1} \rightarrow \Lambda^{i-d} V \otimes\left(I_{X}\right)_{d} \rightarrow \Lambda^{i-d-1} V \otimes\left(I_{X}\right)_{d+1}
$$

where $V=H^{0}\left(\mathcal{O}_{\mathbb{P}^{n-1}}, \mathcal{O}(1)\right)$. Since $d \leq s(X)$ the variety $X$ does not lie on a hypersurface of degree $d-1$ and the first space vanishes. The kernel of the second map is easily identified as the homology group above Ehb94 p. 153].

Remark 3.7. For $d<s(X)$ this lemma only says that the homology groups above vanish. This might seem a trivial observation, but via Proposition 6.1 this will provide a crucial vanishing theorem for the vector bundles used in Section 10.

An interesting class of varieties whose linear strand is even exact is given by certain determinantal varieties:

Definition 3.8. Let $\phi: \mathcal{G} \rightarrow \mathcal{H}$ be a homomorphism of vector bundles on $\mathbb{P}^{n-1}$. Then we define the $r$-th degeneracy locus of $\phi$ by

$$
X_{r}(\phi)=\left\{x \in \mathbb{P}^{n-1} \mid \operatorname{rank} \phi(x) \leq r\right\}
$$

equipped with its natural scheme structure.

Proposition 3.9. Let $G$ and $H$ be vector spaces of dimension $g \geq h$ and $\phi: G \otimes \mathcal{O}(-1) \rightarrow H \otimes \mathcal{O}$ a map whose determinantal locus $X=X_{h-1}(\phi) \subset$ $\mathbb{P}^{n-1}$ is of expected dimension. Then the minimal free resolution

$$
F_{X}^{\bullet} \rightarrow I_{X}
$$

is linear, more precisely $F_{X}^{\bullet}=F^{\bullet}(h)[-h]$. 
Proof. $I_{X}$ is resolved by the Eagon-Northcott complex.

The complexity of minimal free resolutions $F_{X}^{\bullet}$ tends to increase with the codimension of $X$. One approach to understand $F_{X}^{\bullet}$ is therefore to find varieties $X^{\prime} \subset \mathbb{P}^{n-1}$ of smaller codimension that contain $X$. Often their minimal free resolutions contain information about $F_{X}^{\bullet}$ :

Proposition 3.10. Let $X \subset X^{\prime} \subset \mathbb{P}^{n-1}$ be subschemes of $\mathbb{P}^{n-1}$. Assume that $X$ and $X^{\prime}$ have the same postulation $s(X)=s\left(X^{\prime}\right)=: s$. Then there exists a diagram

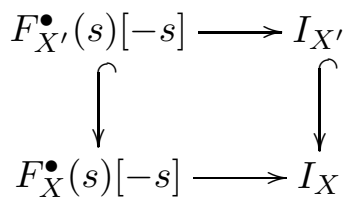

induced by the inclusion $X \hookrightarrow X^{\prime}$.

Proof. Consider the exact sequence

$$
0 \rightarrow I_{X^{\prime}} \rightarrow I_{X} \rightarrow I_{X^{\prime} / X} \rightarrow 0 .
$$

Tensoring with $\Omega^{-i-s}(i)$ defines an inclusion

$$
H^{0}\left(I_{X^{\prime}} \otimes \Omega^{-i-s}(-i)\right) \hookrightarrow H^{0}\left(I_{X} \otimes \Omega^{-i-s}(-i)\right)
$$

which by Lemma 3.6 induces an inclusion

$$
F_{s, X^{\prime}}^{i} \hookrightarrow F_{s, X}^{i} .
$$

This inclusion commutes with the differentials, since these are induced by the natural map

$$
\Omega^{-i-s}(-i) \rightarrow \Omega^{-i-1-s}(-i-1) \otimes H^{0}(\mathcal{O}(1))
$$

for both varieties.

This proposition gives a method to prove the non vanishing of certain syzygy spaces:

Example 3.11 (Green and Lazarsfeld). Let $X \subset \mathbb{P}^{g-1}$ be a canonical curve, and assume that $|D|$ is a pencil of degree $d$ divisors. Consider the vector spaces $G=H^{0}(K-D)$ and $H=H^{0}(D)$ and the map

$$
\phi: G \otimes \mathcal{O}_{\mathbb{P}^{g-1}}(-1) \rightarrow H^{*} \otimes \mathcal{O}_{\mathbb{P}^{g-1}}
$$

induced by the multiplication of sections. The determinantal variety $X^{\prime}=X_{1}(\phi)$ will contain $X$. It is of expected dimension and cut out by quadrics. By the propositions above, the minimal free resolution of $X^{\prime}$ will be a subcomplex of the minimal free resolution of $X$. In particular $\beta_{-(g-d-1), 2}(X) \neq 0$, since $\beta_{-(g-d-1), 2}\left(X^{\prime}\right) \neq 0$. This proves the "easy direction" of Green's conjecture for general canonical curves. 


\section{Geometric Syzygies}

We shall now explain the concept of geometric syzygies. Let $X \subset \mathbb{P}^{n-1}$ be a projective variety of postulation $s$ and $\mathcal{L}$ a line bundle on $X$. This defines a natural vector bundle homomorphism

$$
\phi_{\mathcal{L}}: H^{0}\left(\mathcal{O}(1) \otimes \mathcal{L}^{-1}\right) \otimes \mathcal{O}(-1) \rightarrow H^{0}(\mathcal{L})^{*} \otimes \mathcal{O}
$$

If $u_{1}, \ldots, u_{g}$ is a basis of $H^{0}\left(\mathcal{O}(1) \otimes \mathcal{L}^{-1}\right)$ and $v_{1}, \ldots, v_{h}$ is a basis of $H^{0}(\mathcal{L})$ then $\phi_{\mathcal{L}}$ is given by the matrix $\left(u_{i} v_{j}\right)$. We may assume $g \geq h$, since we can exchange the roles of $\mathcal{L}$ and $\mathcal{O}(1) \otimes \mathcal{L}^{-1}$. Over $X$ this matrix has rank 1 and hence $X$ is contained in $X_{r}\left(\phi_{\mathcal{L}}\right)$ for $r \geq 1$. Notice that $X_{s-1}\left(\phi_{\mathcal{L}}\right)$ has also postulation $s$ as it is defined by $(s \times s)$-minors.

Definition 4.1. An $i$ th linear syzygy $f \in F_{s, X}^{-i}$ is called geometric if there exists a line bundle $\mathcal{L}$ on $X$ such that $f$ is in the image of the map

$$
F_{s, X^{\prime}}^{-i} \rightarrow F_{s, X}^{-i}
$$

induced by the inclusion $X \hookrightarrow X^{\prime}:=X_{s-1}\left(\phi_{\mathcal{L}}\right)$.

Now consider the special case where $h:=h^{0}(\mathcal{L})$ is equal to the postulation $s$. Then $X_{s-1}\left(\phi_{\mathcal{L}}\right)$ is cut out by the maximal minors. Since $\left(u_{i} v_{j}\right)$ is a $1-$ generic matrix (see for example [Eis88] ) the scheme $X_{s-1}\left(\phi_{\mathcal{L}}\right)$ has expected codimension [Eis88, Corollary 3.3], and hence its minimal free resolution is given by the Eagon-Northcott complex. The geometric interpretation of $X_{s-1}\left(\phi_{\mathcal{L}}\right)$ is that set theoretically

$$
X_{s-1}\left(\phi_{\mathcal{L}}\right)=\bigcup_{D \in|\mathcal{L}|} \operatorname{span}(D) \subset \mathbb{P}^{n-1}
$$

We note at this point that the 1-genericity of $\left(u_{i} v_{j}\right)$ also implies that $X_{s-1}\left(\phi_{\mathcal{L}}\right)$ is smooth outside of $X_{s-2}\left(\phi_{\mathcal{L}}\right)$ which is at least of codimension 2 Eis88, Corollary 3.3]. As a determinantal variety of expected codimension $X_{s-1}\left(\phi_{\mathcal{L}}\right)$ is Cohen-Macaulay and together with the above this shows that it is normal.

Definition 4.2. A syzygy $f \in F_{s, X}^{-i}$ is called scrollar, if there exsists a line bundle $\mathcal{L}$ on $X$ with $h^{0}(\mathcal{L})=s$ such that $f$ is geometric with respect to $\mathcal{L}$.

Definition 4.3. We will say that $X$ satisfies the geometric syzygy conjecture in step $i$, if $F_{s, X}^{-i}$ is spanned by geometric syzygies.

The aim of our paper will be to prove the geometric syzygy conjecture for elliptic normal curves and their secant varieties. As a corollary we also obtain the geometric syzygy conjecture for bielliptic canonical curves.

Since one line bundle produces rarely enough syzygies to span those of $X$, we will consider families of line bundles, the determinantal varieties associated to them, and the corresponding families of linear strands. 


\section{FAMilies OF LineAR STRANDS}

In this section we will explore the above concepts in a relative setting. Let

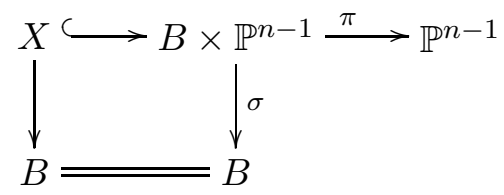

be a family of projective schemes over a smooth, complete and irreducible base $B$. We shall assume that the postulation $s\left(X_{b}\right)$ of the fiberes has constant value $s$ for all $b \in B$.

Definition 5.1. A complex $\mathcal{F}^{\bullet}(s)$ of the form

$$
\mathcal{F}^{\bullet}(s): \cdots \rightarrow \mathcal{F}^{-s-1} \otimes \mathcal{O}(-s-1) \rightarrow \mathcal{F}^{-s} \otimes \mathcal{O}(-s)
$$

with $\mathcal{F}^{i}$ vector bundles on $B$ together with a map

$$
\mathcal{F}^{\bullet}(s)[-s] \rightarrow I_{X}
$$

is called a family of (minimal degree) linear strands, if

$$
\mathcal{F}^{\bullet}(s)[-s] \otimes \mathcal{O}_{\mathbb{P}_{b}^{n-1}} \rightarrow I_{X_{b}}
$$

is the minimal degree linear stand of $X_{b}$ for all $b \in B$.

If $\mathcal{F}^{\bullet}(s)[-s] \rightarrow I_{X}$ is exact one can calculate the terms of $\mathcal{F}^{\bullet}$ by a relative version of Koszul cohomology:

Lemma 5.2. If $\mathcal{F}^{\bullet}(s)[-s] \rightarrow I_{X}$ is an exact family of linear strands, then

$$
\mathcal{F}^{-i}=\sigma_{*}\left(I_{X} \otimes \pi^{*} \Omega^{i-s}(i)\right)
$$

and all higher direct images vanish, i.e.

$$
R^{q} \sigma_{*}\left(I_{X} \otimes \pi^{*} \Omega^{i-s}(i)\right)=0
$$

for $q \geq 1$.

Proof. We use hypercohomology with respect to $\sigma$. The second hypercohomology spectral sequence is

$$
{ }^{I I} E_{2}^{p q}=R^{p} \sigma_{*}\left(H^{q}\left(\mathcal{F}^{\bullet}\right)\right) \Rightarrow \mathbb{H}_{\sigma}^{p+q}\left(\mathcal{F}^{\bullet}\right) .
$$

Since $\mathcal{F}(s)[-s]$ has cohomology $I_{X}$ concentrated in degree 0 we have

$$
\begin{aligned}
{ }^{I I} E_{2}^{p q} & =R^{p} \sigma_{*}\left(H^{q}\left(\mathcal{F}^{\bullet}(s)[-s] \otimes \pi^{*} \Omega^{i-s}(i)\right)\right) \\
& =\left\{\begin{array}{cc}
R^{p} \sigma_{*}\left(I_{X} \otimes \pi^{*} \Omega^{i-s}(i)\right) & \text { if } q=0 \\
0 & \text { otherwise }
\end{array}\right.
\end{aligned}
$$

i.e. the spectral sequence degenerates and

$$
\mathbb{H}_{\sigma}^{p}:=\mathbb{H}_{\sigma}^{p}\left(\mathcal{F}^{\bullet}(s)[-s] \otimes \pi^{*} \Omega^{i-s}(i)\right)=R^{p} \sigma_{*}\left(I_{X} \otimes \pi^{*} \Omega^{i-s}(i)\right) .
$$

To prove the identities of the lemma we use the first hypercohomology sequence

$$
{ }^{I} E_{1}^{p q}=\left(R^{q} \sigma_{*}\left(\mathcal{F}^{\bullet}\right)\right)_{p} \Rightarrow \mathbb{H}_{\sigma}^{p+q}\left(\mathcal{F}^{\bullet}\right) .
$$

We start by calculating the higher direct images of $\mathcal{F}^{\bullet}(s)[-s] \otimes \pi^{*} \Omega^{i-s}(i)$ in step $p$. By Hilbert's syzygy theorem $\mathcal{F}^{\bullet}(s)[-s]$ has no non zero terms 
in steps $p \leq-n$. Similariy by definition it has no non zero terms in steps $p>0$. For $-n-1 \leq p \leq 0$ we have

$$
\begin{aligned}
{ }^{I} E_{1}^{p q} & =R^{q} \sigma_{*}\left[\mathcal{F}^{\bullet}(s)[-s] \otimes \pi^{*} \Omega^{i-s}(i)\right]_{p} \\
& =R^{q} \sigma_{*}\left(\mathcal{F}^{p-s}(p-s) \otimes \pi^{*} \Omega^{i-s}(i)\right) \\
& =\mathcal{F}^{p-s} \otimes R^{q} \sigma_{*} \pi^{*} \Omega^{i-s}(i-s+p) .
\end{aligned}
$$

By Lemma 13.1 in the appendix the second factor is non zero only for $p=-i+s=-q$. Furthermore $R^{i-s} \sigma_{*} \pi^{*} \Omega^{i-s}$ is trivial. So

$$
{ }^{I} E_{1}^{p q}=\left\{\begin{array}{cc}
\mathcal{F}^{-i} & \text { if } p=-i+s=-q \\
0 & \text { otherwise. }
\end{array}\right.
$$

So this spectral sequence collapses also and gives

$$
\mathbb{H}_{\sigma}^{p+q}=\left\{\begin{array}{cc}
\mathcal{F}^{-i} & \text { if } p+q=0 \\
0 & \text { otherwise. }
\end{array}\right.
$$

Comparing this with our previous calculation completes the proof.

Example 5.3. The most important exact families of linear strands are the Eagon-Northcott complexes associated to maps of vector bundles

$$
\phi: \mathcal{G} \otimes \mathcal{O}(-1) \rightarrow \mathcal{H} \otimes \mathcal{O}
$$

of rank $g \geq h$ such that $X=X_{h}(\phi)$ is of expected dimension on every fiber $X_{b}$. The $X_{b}$ are determinantal varieties as in proposition 3.9.

We now want to relate the minimal degree linear strands of a family to those of the scheme theoretic intersection $\cap X:=\bigcap_{b \in B} X_{b}$ and the scheme theoretic union $\cup X:=\bigcup_{b \in B} X_{b}$ of the fibres of $X$. Suppose that $\cap X, \cup X$ and $X_{b}$ all have the same postulation $s$. Then by Proposition 3.10 the minimal degree linear strand of $\cup X$ is a subcomplex of all minimal degree linear strands in the family, while all minimal degree linear strands of the family are subcomplexes of the minimal degree linear strand of $\cap X$

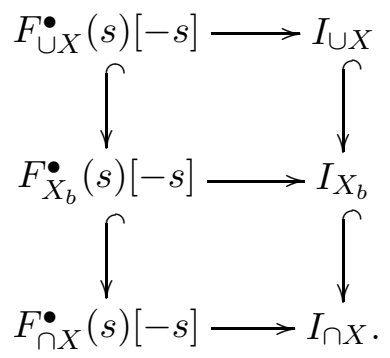

A natural question is, wether we can construct the minimal degree linear strands $F_{\cup_{X}}^{\bullet}(s)$ and $F_{\cap X}^{\bullet}(s)$ from the family $\mathcal{F}^{\bullet}(s)$.

Remark 5.4. If $X$ is a family of determinantal varieties obtained from a familiy of line bundles as in Section 4 the images of each inclusion

$$
F_{X_{b}}^{\bullet}(s)[-s] \hookrightarrow F_{\cap X}^{\bullet}(s)[-s]
$$

will consist of geometric syzygies. 


\section{The UNION OF FIBRES}

We shall consider the union

$$
\cup X:=\bigcup_{b \in B} X_{b}
$$

with the natural scheme structure given by

$$
I_{\cup X}:=\pi_{*} I_{X} .
$$

Appling the functor $\pi_{*}$ to the family of linear strands

$$
\mathcal{F}^{\bullet}(s)[-s] \rightarrow I_{X}
$$

we obtain a complex

$$
\pi_{*} \mathcal{F}^{\bullet}(s)[-s] \rightarrow I_{\cup X}
$$

with

$$
\pi_{*} \mathcal{F}^{\bullet}(s): \cdots \rightarrow H^{0}\left(B, \mathcal{F}^{-s-1}\right) \otimes \mathcal{O}(-s-1) \rightarrow H^{0}\left(B, \mathcal{F}^{-s}\right) \otimes \mathcal{O}(-s) .
$$

This complex is often the minimal degree linear strand of $\cup X$ :

Proposition 6.1. Let $\mathcal{F}^{\bullet}(s)[-s] \rightarrow I_{X}$ be an exact family of linear strands with $\mathcal{F}^{-i}=0$ for $i>s+n$. If $\cup X$ has the same postulation $s$, then its minimal degree linear strand is

$$
\pi_{*} \mathcal{F}^{\bullet}(s)[-s] \rightarrow I \cup X
$$

otherwise $\pi_{*} \mathcal{F}^{\bullet}(s)$ vanishes.

Proof. Since no fiber $X_{b}$ lies on a hypersurface of degree smaller than $s$, the same is true for the union $\cup X$. We can therefore calculate the degree $s$ linear strand of $\cup X$ via Lemma 3.6 and 5.2

$$
\begin{aligned}
F_{s, \cup X}^{-i} & =H^{0}\left(\mathbb{P}^{n-1}, I_{\cup X} \otimes \Omega^{i-s}(i)\right) \\
& =H^{0}\left(\mathbb{P}^{n-1} \times B, I_{X} \otimes \pi^{*} \Omega^{i-s}(i)\right) \\
& =H^{0}\left(B, \sigma_{*}\left(I_{X} \otimes \pi^{*} \Omega^{i-s}(i)\right)\right. \\
& =H^{0}\left(B, \mathcal{F}^{-i}\right)
\end{aligned}
$$

If the postulation of $\cup X$ is larger than $s$, its degree $s$ linear strand is zero.

\section{The INTERSECTION OF FIBRES}

The linear strand of the intersection of fibres is much harder to control. For this we consider functor

$$
R:=R^{\operatorname{dim} B} \pi_{*}\left(-\otimes \sigma^{*} \omega_{B}\right)
$$

where $\omega_{B}$ is the canonical sheaf on $B$. $R$ is right exact since the fibres of $\pi$ have dimension $\operatorname{dim} B$.

We start with the following lemma. 
Lemma 7.1. Let $\mathcal{F}^{\bullet}(s)$ be a family of (minimal degree) linear strands such that

$$
\mathcal{F}^{\bullet}(s)[-s+1] \rightarrow \mathcal{O}_{B \times \mathbb{P}^{n-1}} \rightarrow \mathcal{O}_{X} \rightarrow 0
$$

is an exact sequence. Then there is a natural isomorphism

$$
\text { tr : } R \mathcal{O}_{X} \cong \mathcal{O}_{\cap X} \text {. }
$$

Proof. Since

$$
\mathcal{F}^{\bullet}(s)[-s+1] \rightarrow \mathcal{O}_{B \times \mathbb{P}^{n-1}} \rightarrow \mathcal{O}_{X} \rightarrow 0
$$

is exact and restriction to the fibres $X_{b}$ is right exact, we obtain an exact sequence

$$
\mathcal{F}_{b}^{-s} \otimes \mathcal{O}(-s) \rightarrow \mathcal{O}_{\mathbb{P}_{b}^{n-1}} \rightarrow \mathcal{O}_{X_{b}} \rightarrow 0
$$

I.e. $I_{X_{b}}$ is generated by the image of $\mathcal{F}_{b}^{-s} \otimes \mathcal{O}(-s)$. On the other hand applying $R$ yields the exact sequence

$$
H^{\operatorname{dim} B}\left(\mathcal{F}^{-s} \otimes \omega_{B}\right) \otimes \mathcal{O}(-s) \rightarrow H^{\operatorname{dim} B}\left(\omega_{B}\right) \otimes \mathcal{O}_{\mathbb{P}^{n-1}} \rightarrow R \mathcal{O}_{X} \rightarrow 0 .
$$

We shall now compare the two exact sequences. For every point $b \in B$ restriction defines a map

$$
H^{0}\left(B,\left(\mathcal{F}^{-s}\right)^{*}\right) \rightarrow H^{0}\left(B,\left(\mathcal{F}_{b}^{-s}\right)^{*}\right) .
$$

Dualizing this gives a homomorphism

$$
\mathcal{F}_{b}^{-s} \rightarrow H^{\operatorname{dim} B}\left(B, \mathcal{F}^{-s} \otimes \omega_{B}\right)=H^{0}\left(R \mathcal{F}^{-s}\right) .
$$

Similarly we obtain

$$
\mathcal{O}_{\mathbb{P}_{b}^{n-1}} \rightarrow H^{\operatorname{dim} B}\left(\omega_{B}\right)
$$

Since the image of $\mathcal{F}_{b}^{-s} \otimes \mathcal{O}(-s)$ generates $H^{\operatorname{dim} B}\left(\omega_{B}\right) \otimes I_{X_{b}}$, the image of $H^{\operatorname{dim} B}\left(\mathcal{F}^{-s} \otimes \omega_{B}\right) \otimes \mathcal{O}(-s)$ equals $H^{\operatorname{dim} B}\left(\omega_{B}\right) \otimes I_{\cap X}$ and hence the epimorphism

$$
H^{\operatorname{dim} B}\left(\omega_{B}\right) \otimes \mathcal{O}_{\mathbb{P}^{n-1}} \rightarrow R \mathcal{O}_{X} \rightarrow 0
$$

induces an isomorphism

$$
H^{\operatorname{dim} B}\left(\omega_{B}\right) \otimes \mathcal{O}_{\cap X} \rightarrow R \mathcal{O}_{X} \rightarrow 0 .
$$

Since $H^{\operatorname{dim} B}\left(\omega_{B}\right)$ is 1-dimensional this proves the lemma.

Remark 7.2. Duality theory defines a trace homomorphism

$$
\operatorname{tr}: R \mathcal{O}_{X} \rightarrow \mathcal{O}_{\cap X} \rightarrow 0 \text {. }
$$

In this situation this is (up to possibly a scalar) nothing but the isomorphism from the above lemma. For this reason we already denoted the isomorphism of the lemma by tr.

So if we apply $R$ to the complex

$$
\mathcal{F}^{\bullet}(s)[-s+1] \rightarrow \mathcal{O}_{B \times \mathbb{P}^{n-1}} \rightarrow \mathcal{O}_{X}
$$

we obtain a complex

$$
R \mathcal{F}^{\bullet}(s)[-s+1] \rightarrow \mathcal{O} \rightarrow \mathcal{O}_{\cap X}
$$

where

$$
\left(R \mathcal{F}^{\bullet}\right)_{i}=H^{\operatorname{dim} B}\left(\mathcal{F}_{i} \otimes \omega_{B}\right) \otimes \mathcal{O}(-i-d)=H^{0}\left(\mathcal{F}_{i}^{*}\right)^{*} \otimes \mathcal{O}(-i-d)
$$


unfortunately it is not so easy to determine, whether this is the linear strand of $\cap X$. We shall see in Remark 10.8 and more detailed in Section 11] that this is not always the case.

Still we have

Proposition 7.3. Assume $s=s\left(X_{b}\right)=s(\cap X)$ and let $F_{\cap X}^{\bullet}(s)[-s] \rightarrow I_{\cap X}$ be the minimal degree linear strand of $\cap X$. Then there is a natural map of complexes

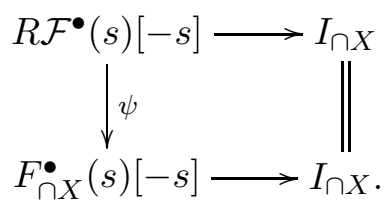

Proof. Consider the minimal free resolution

$$
F_{\cap X}^{\bullet} \rightarrow I_{\cap X}
$$

of $\cap X$. Since

$$
R \mathcal{F}^{\bullet}(s)[-s] \rightarrow I_{\cap X}
$$

is projective, the identity lifts to a map of complexes

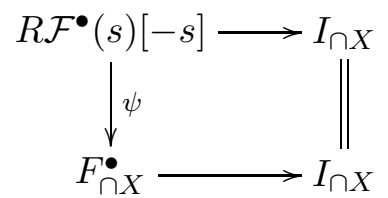

which is unique up to homotopy equivalence (see for example Wei94, p. 35, Porism 2.2.7]). For degree reasons the image of $\psi$ lies in the minimal degree linear strand

$$
F_{\cap X}^{\bullet}(s)[-s] \rightarrow I_{\cap X} .
$$

Once again by degree reasons there exists no non trivial homotopy equivalence in this situation.

Remark 7.4. If $X$ is a family of determinantal varieties obtained from a family of line bundles as in Section 4 , then the image of $\psi$ is the part of the minimal free resolution of $\cap X$ that is spanned by geometric syzygies coming from this family. Our plan is to find a family $X$ such that $\cap X=\operatorname{Sec}_{d-1}$ and $\psi$ surjective.

Before we can do this, we first construct the minimal free resolution of $\mathrm{Sec}_{d-1}$ as a mapping cone.

\section{THE Minimal FREe RESOLUtion OF $\mathrm{Sec}_{d-1}$}

In this section we construct a minimal free resolution of the secant variety $\operatorname{Sec}_{d-1}$ of $E$. The main idea is to find a scroll $X_{|D|}$ that contains $\operatorname{Sec}_{d-1}$ such that the ideal sheaf $I_{\operatorname{Sec}_{d-1} / X_{|D|}}$ is the dualizing sheaf of $X_{|D|}$. In this situation a minimal free resolution for $I_{\operatorname{Sec}_{d-1} / X_{|D|}}$ is given by the dual of the minimal free resolution of $X_{|D|}$ which is itself just an Eagon-Northcott 
complex. The minimal free resolution of $\mathcal{O}_{\operatorname{Sec}_{d-1}}$ is then obtained by a mapping cone construction from these two resolutions. We learned this method from Sch86.

Let $\mathcal{L}=\mathcal{O}_{E}(D)$ where $D$ is a divisor on $E$ of degree $d$. From now on we shall assume $d \leq \frac{n}{2}$. The multiplication map

$$
\mu: H^{0}(n A-D) \otimes H^{0}(D) \rightarrow H^{0}(n A)
$$

induces a map of vector bundles

$$
\phi_{\mathcal{L}}: H^{0}(n A-D) \otimes \mathcal{O}(-1) \rightarrow H^{0}(D)^{*} \otimes \mathcal{O}
$$

on $\mathbb{P}^{n-1}$. As in Section 4 this defines the scroll

$$
X_{|D|}:=X_{d-1}\left(\phi_{\mathcal{L}}\right)=\bigcup_{D^{\prime} \in|D|} \operatorname{span}\left(D^{\prime}\right) \subset \mathbb{P}^{n-1}
$$

On the projective space $\mathbb{P}\left(H^{0}(D)^{*}\right) \cong \mathbb{P}^{d-1}$ the multiplication $\mu$ induces an injective map $\tilde{\mu}$ with cokernel $\mathcal{E}$ :

$$
0 \rightarrow H^{0}(n A-D) \otimes \mathcal{O}_{\mathbb{P}^{d-1}}(-1) \stackrel{\tilde{\mu}}{\rightarrow} H^{0}(n A) \otimes \mathcal{O}_{\mathbb{P}^{d-1}} \stackrel{\rho}{\rightarrow} \mathcal{E} \rightarrow 0
$$

Let $\tau: \mathbb{P}(\mathcal{E}) \rightarrow \mathbb{P}^{d-1}$ be the structure map. $X_{|D|}$ is the image of the projectivization of $\rho$

$$
\rho: \mathbb{P}(\mathcal{E}) \rightarrow \mathbb{P}^{n-1}
$$

Notice that $\mathbb{P}(\mathcal{E})$ is the canonical desingularisation of the determinantal variety $X_{|D|}$ given by

$$
\mathbb{P}(\mathcal{E})=\left\{(x, y) \in \mathbb{P}^{n-1} \times \mathbb{P}^{d-1} \mid y \circ \phi_{x}=0\right\}
$$

where $\phi_{x}: H^{0}(n A-D) \rightarrow H^{0}(D)^{*}$ is restriction of $\phi$ to $x$ and $y$ is viewed as a linear form $y: H^{0}(D)^{*} \rightarrow \mathbb{C}$.

For future calculations we will denote by $H$ the pullback of the hyperplane class of $\mathbb{P}^{n-1}$ to $\mathbb{P}(\mathcal{E})$, i.e. $\mathcal{O}_{\mathbb{P}(\mathcal{E})}(H)=\mathcal{O}_{\mathbb{P}(\mathcal{E})}(1)$. Let $R$ be the preimage of a hyperplane of $\mathbb{P}^{d-1}$.

Remark 8.1. $X_{|D|}$ contains $\operatorname{Sec}_{d-1}$ since for every set of points $p_{1}, \ldots, p_{d-1}$ we have

$$
H^{0}\left(D-p_{1}-\cdots-p_{d-1}\right)=1
$$

i.e there exist a unique fiber of $\mathbb{P}(\mathcal{E})$ whose image contains the secant through $p_{1}, \ldots, p_{d-1}$. Moreover there is only one such fiber.

We want to show, that the preimage

$$
\widetilde{\operatorname{Sec}_{d-1}}=\rho^{-1} \operatorname{Sec}_{d-1}
$$

and $K_{\mathbb{P}(\mathcal{E})}$ have the same class. For this we first calculate some intersection numbers.

Lemma 8.2. Let $X=X_{l-1}(\phi)$ be a determinantal variety defined by the maximal minors of a $k \times l$-matrix $(k \geq l)$ of linear forms. If $X$ has the expected codimension $k$ then

$$
\operatorname{deg} X=\left(\begin{array}{c}
k \\
l-1
\end{array}\right)
$$


Proof. Since $X$ is a linear section of the generic determinantal variety $M_{l-1}(k, l)$ and of expected dimension, both varieties have the same degree. This degree is calculated in [ACGH85, p. 95] as

$$
\begin{aligned}
\operatorname{deg}\left(M_{l-1}(k, l)\right) & =\Pi_{i=0}^{l-(l-1)-1} \frac{(k+i) ! i !}{(l-1+i) !(k-(l-1)+i) !} \\
& =\frac{k !}{(l-1) !(k-l+1) !} \\
& =\left(\begin{array}{c}
k \\
l-1
\end{array}\right) .
\end{aligned}
$$

Corollary 8.3. On $\mathbb{P}(\mathcal{E})$ we have the following intersection numbers

$$
H^{2 d-i-2} R^{i}=\left(\begin{array}{c}
n-d \\
d-i-1
\end{array}\right)
$$

Proof. The class $R^{i}$ is realized by the preimage of a codimension $i$ linear subspace $\mathbb{P}^{d-i-1} \subset \mathbb{P}^{d}$. Its image in $X_{|D|}$ is defined by the maximal minors of the corresponding $(n-d) \times(d-i)$-submatrix of $H^{0}(n A-D) \otimes H^{0}(D) \rightarrow$ $H^{0}(n A)$. By the lemma above its degree is

$$
H^{2 d-i-2} R^{i}=\operatorname{deg} \pi^{*} \mathbb{P}^{d-i-1}=\left(\begin{array}{c}
n-d \\
d-i-1
\end{array}\right) .
$$

This allows us to calculate the class and degree of the canonical divisor on $\mathbb{P}(\mathcal{E})$ :

Proposition 8.4. The canonical divisor $K_{\mathbb{P}(\mathcal{E})}$ has class

$$
K_{\mathbb{P}(\mathcal{E})}=-d H+(n-2 d) R
$$

and degree

$$
H^{2 d-1} K_{\mathbb{P}(\mathcal{E})}=-\left(\begin{array}{c}
n-d+1 \\
d-1
\end{array}\right)-\left(\begin{array}{l}
n-d \\
d-2
\end{array}\right)
$$

Proof. The relative canonical sheaf of $\mathbb{P}(\mathcal{E})$ is

$$
\omega_{\mathbb{P}(\mathcal{E}) / \mathbb{P}^{d-1}}=\tau^{*}\left(\Lambda^{d} \mathcal{E}\right)(-d H)=\mathcal{O}(-d H+(n-d) R)
$$

by the defining sequence of the vector bundle $\mathcal{E}$. On the other hand $\tau^{*}\left(\omega_{\mathbb{P}^{d-1}}\right)=\mathcal{O}(-d R)$, and therefore $K_{\mathbb{P}(\mathcal{E})}=-d H+(n-2 d) R$.

The degree of $K_{\mathbb{P}(\mathcal{E})}$ is

$$
\begin{aligned}
H^{2 d-1} K_{\mathbb{P}(\mathcal{E})} & =-d H^{2 d-2}+(n-2 d) H^{2 d-3} R \\
& =-d\left(\begin{array}{c}
n-d \\
d-1
\end{array}\right)+(n-2 d)\left(\begin{array}{c}
n-d \\
d-2
\end{array}\right) \\
& =-\left(\begin{array}{c}
n-d+1 \\
d-1
\end{array}\right)-\left(\begin{array}{c}
n-d \\
d-2
\end{array}\right) .
\end{aligned}
$$


Proposition 8.5. Let $d<\frac{n}{2}+1$ then the degree of the secant-variety $\operatorname{Sec}_{d-1} E$ is

$$
\operatorname{deg} \operatorname{Sec}_{d-1} E=\left(\begin{array}{c}
n-d \\
d-2
\end{array}\right)+\left(\begin{array}{c}
n-d+1 \\
d-1
\end{array}\right)
$$

Proof. The codimension of $\operatorname{Sec}_{d-1} E \subset \mathbb{P}^{n-1}$ is $n-2 d+2$. If we choose a general $n-2 d+2$-dimensional linear subspace of $\mathbb{P}^{n-1}$ it intersects $\operatorname{Sec}_{d-1} E$ in $\operatorname{deg} \operatorname{Sec}_{d-1} E$ points. Now project from this subspace. We obtain a degree $n$ elliptic curve in $\mathbb{P}^{2 d+4}$ whose number of $(d-1)$-secant $\mathbb{P}^{d-3}$ 's is exactly $\operatorname{deg} \operatorname{Sec}_{d-1} E$. This number $v_{d-2}$ is calculated in [ACGH85, p. 351]:

$$
\begin{aligned}
v_{d-2} & =\sum_{\alpha=0}^{d-1}(-1)^{\alpha}\left(\begin{array}{c}
1+2(d-2)-n \\
\alpha
\end{array}\right)\left(\begin{array}{c}
1 \\
d-1-\alpha
\end{array}\right) \\
& =(-1)^{d-2}\left(\begin{array}{c}
2 d-3-n \\
d-2
\end{array}\right)+(-1)^{d-1}\left(\begin{array}{c}
2 d-3-n \\
d-1
\end{array}\right) \\
& =\left(\begin{array}{c}
n-d \\
d-2
\end{array}\right)+\left(\begin{array}{c}
n-d+1 \\
d-1
\end{array}\right) .
\end{aligned}
$$

Corollary 8.6. The preimage $\widetilde{\operatorname{Sec}_{d-1}}$ of the secant variety $\operatorname{Sec}_{d-1}$ is an anticanonical divisor on $\mathbb{P}(\mathcal{E})$.

Proof. Since $\widetilde{\operatorname{Sec}_{d-1}}$ is a divisor of $\mathbb{P}(\mathcal{E})$ it has class $a H+b R$, for some integers $a$ and $b$ to be determined.

By the Propositions 8.4 and 8.5 we have

$$
H^{2 d-3} \widetilde{\operatorname{Sec}_{d-1}}=-H^{2 d-3} K_{\mathbb{P}(\mathcal{E})}
$$

and $K_{\mathbb{P}(\mathcal{E})}=-d H+(n-2 d) R$. So it is enough to show, that $a=d$ :

The image of a fiber of $\mathbb{P}(\mathcal{E})$ in $\mathbb{P}^{n-1}$ contains $d$ points of $E$ and therefore $d$ distinct $(d-1)$-secant $(d-2)$-planes. Conversely the intersection of any other $d$-1-secant $(d-2)$-plane with the image of a fiber of $\mathbb{P}(\mathcal{E})$ which is a $d$-secant $(d-1)$-plane is spanned by their common secant points (see Lemma 13.2 in the appendix), and this span is contained in one of the $(d-1)$-secants above. This shows

$$
\left(\widetilde{\operatorname{Sec}_{d-1}} E\right) \cdot R^{d-1}=d H R^{d-1}
$$

and consequently $a=d$.

Lemma 8.7. Let $F_{|D|}^{\bullet}[1] \rightarrow \mathcal{O} \rightarrow \mathcal{O}_{X_{|D|}}$ be the minimal free resolution of $X_{|D|}$, then $F_{|D|}^{\bullet}=F_{|D|}^{\bullet}(d)[-d]=E N^{\bullet}(\phi)[-d]$ where

$$
E N^{-i}(\phi)=\Lambda^{i} H^{0}(n A-D) \otimes \Lambda^{d} H^{0}(D) \otimes S_{i-d} H^{0}(D) \otimes \mathcal{O}(-i)
$$

is the Eagon-Northcott complex associated to $\phi$.

Proof. Since $X_{|D|}$ is the locus where $\phi$ drops rank, and since $X_{|D|}$ is of expected dimension, the Eagon-Northcott complex is a minimal free resolution. 
Example 8.8. Let $n=6$. Then the Betti diagram of $\mathcal{O}_{X_{|D|}}$ is

$$
\begin{aligned}
& \begin{array}{cccc}
- & - & - & 1 \\
3 & 8 & 6 & -
\end{array} \text { and } \quad \begin{array}{cc}
- & 1 \\
- & - \\
1 & -
\end{array} \\
& \text { if } \operatorname{deg} D=2 \quad \text { if } \operatorname{deg} D=3 .
\end{aligned}
$$

Before we can prove the main result of this section, we need a technical lemma.

Lemma 8.9. If $\omega_{X_{|D|}}$ is the dualizing sheaf of $X_{|D|}$ then

$$
I_{\operatorname{Sec}_{d-1} / X_{|D|}} \cong \omega_{X|D|}
$$

Proof. Let $U$ be the regular part of $X_{|D|}$. Since the singular locus of $X_{|D|}$ contains the locus where $\rho$ has positive dimensional fibres, the restriction of $\rho$ to $\rho^{-1}(U)$ defines an isomorphism of $\rho^{-1}(U)$ with $U$. Hence by the above Corrolary [8.6] we find that

$$
I_{\operatorname{Sec}_{d-1} \cap U / U}=\omega_{U}
$$

Pushing this isomorphism forward with the inclusion

$$
i: U \rightarrow X_{|D|}
$$

gives the result since $X_{|D|}$ is normal as seen in Section 4

We have now all the necessary ingredients to prove the main result of this section:

Theorem 8.10. Let $F_{\operatorname{Sec}_{d-1}}^{\bullet} \rightarrow I_{\operatorname{Sec}_{d-1}}$ be the minimal free resolution of the $d-1$-secant variety of $E$, and $D$ any divisor of degree $d \leq n / 2$. Then the minimal free resolution of $\mathcal{O}_{\mathrm{Sec}_{d-1}}$ is

$$
\mathcal{O}(-n) \rightarrow F_{\operatorname{Sec}_{d-1}}^{\bullet}(d)[-d+1] \rightarrow \mathcal{O} \rightarrow \mathcal{O}_{\operatorname{Sec}_{d-1}}
$$

and there exist an exact sequence

$$
0 \rightarrow F_{|D|}^{\bullet}(d) \rightarrow F_{\operatorname{Sec}_{d-1}}^{\bullet}(d) \rightarrow F_{|D|}^{\bullet}(d)^{*} \rightarrow 0
$$

where $F_{|D|}(d)^{*}:=\mathcal{H o m}\left(F_{|D|}^{\bullet}(d), \omega_{\mathbb{P}^{n-1}}\right)[n]$.

Proof. By Lemma 8.9 above we have $I_{\operatorname{Sec}_{d-1} / X_{|D|}}=\omega_{X_{|D|}}$. If we apply $\mathcal{H o m}\left(-, \omega_{\mathbb{P}^{n-1}}\right)[n]$ to the minimal free resolution

$$
F_{|D|}^{\bullet}(d)[-d+1] \rightarrow \mathcal{O} \rightarrow \mathcal{O}_{X_{|D|}}
$$

we obtain an exact sequence

$$
\mathcal{O}(-n) \rightarrow F_{|D|}^{\bullet}(d)^{*}[-d] \rightarrow \omega_{X|D|}
$$

i.e. a free resolution of $\omega_{X_{|D|}}$.

Now consider the short exact sequence

$$
0 \rightarrow I_{X_{|D|}} \rightarrow I_{\operatorname{Sec}_{d-1}} \rightarrow I_{\operatorname{Sec}_{d-1} / X_{|D|}} \rightarrow 0 .
$$

By the horseshoe lemma the direct sum of the two minimal free resolutions of $I_{X_{|D|}}$ and $I_{\operatorname{Sec}_{d-1} / X_{|D|}}$ fits together to form a (not necessarily minimal) free resolution of the middle term $I_{\mathrm{Sec}_{d-1}}$. For degree reasons this resolution 
is minimal. This proves that the minimal free resolution of $\mathcal{O}_{\operatorname{Sec}_{d-1}}$ has the shape claimed and that for the linear strands of all three resolutions in this construction the claimed exact sequence hold.

Remark 8.11. In the case $d=(n+1) / 2$ the variety $\operatorname{Sec}_{d-1}$ is a hypersurface of degree $n$. Here the analog of Theorem 8.10 still holds. The minimal free resolution is given by

$$
\mathcal{O}(-n) \rightarrow \mathcal{O} \rightarrow \mathcal{O}_{\mathrm{Sec}_{d}}
$$

Example 8.12. Let $n=6$. Then we obtain the Betti diagram of $\mathcal{O}_{E}=$ $\mathcal{O}_{\mathrm{Sec}_{1} E}$ via

$$
\begin{array}{ccccccccccccccccccc}
- & - & - & - & 1 & & - & - & - & - & - & & - & - & - & - & 1 \\
- & 3 & 8 & 6 & - & + & - & 6 & 8 & 3 & - & = & - & 9 & 16 & 9 & - \\
- & - & - & - & - & & 1 & - & - & - & - & & 1 & - & - & - & -
\end{array}
$$

Similarly we obtain the Betti diagram of $\mathcal{O}_{\mathrm{Sec}_{2} E}$ via

$$
\begin{array}{lllllllllll}
- & - & 1 & & - & - & - & & - & - & 1 \\
- & - & - & & - & - & - & & - & - & - \\
- & 1 & - & + & - & 1 & - & = & - & 2 & - \\
- & - & - & & - & - & - & & - & - & -
\end{array}
$$

The above theorem allows us to give closed formulae for the graded Betti numbers of $\operatorname{Sec}_{d-1}$ :

Corollary 8.13. $\beta_{-i d}=\operatorname{dim}\left(F_{\operatorname{Sec}_{d-1}}^{-i}\right)_{d}=\left(\begin{array}{c}n-d \\ i\end{array}\right)\left(\begin{array}{c}i-1 \\ i-d\end{array}\right)+\left(\begin{array}{c}n-d \\ n-i\end{array}\right)\left(\begin{array}{c}n-i-1 \\ n-i-d\end{array}\right)$.

Proof.

$$
\begin{aligned}
\operatorname{dim}\left(F_{\text {Sec }_{d-1}}^{-i}\right)_{d} & =\operatorname{dim} E N^{-i}+\operatorname{dim} E N^{n-i} \\
& =\left(\begin{array}{c}
n-d \\
i
\end{array}\right)\left(\begin{array}{c}
i-1 \\
i-d
\end{array}\right)+\left(\begin{array}{c}
n-d \\
n-i
\end{array}\right)\left(\begin{array}{c}
n-i-1 \\
n-i-d
\end{array}\right)
\end{aligned}
$$

Corollary 8.14. Let $d \leq(n+1) / 2$. Then $\mathrm{Sec}_{d-1}$ is an arithmetically Gorenstein variety. Moreover the dualizing sheaf of $\mathrm{Sec}_{d-1}$ is trivial

$$
\omega_{\mathrm{Sec}_{d-1}}=\mathcal{O}_{\mathrm{Sec}_{d-1}}
$$

Proof. By the Auslander-Buchsbaum formula we can read of the depth of the coordinate ring of $\mathrm{Sec}_{d-1}$ from the minimal free resolution. Since this is the same as the codimension of $\operatorname{Sec}_{d-1}$ in $\mathbb{P}^{n-1}$ this shows that $\operatorname{Sec}_{d-1}$ is arithmetically Cohen-Macaulay.

By applying $\mathcal{H o m}\left(-, \omega_{\mathbb{P}^{n-1}}\right)[n]$ to the minimal free resolution of $\mathcal{O}_{\mathrm{Sec}_{d-1}}$ we obtain a minimal free resolution of $\omega_{\mathrm{Sec}_{d-1}}$. By the Theorem 8.10 and the remark following it the minimal free resolution of $\mathrm{Sec}_{d-1}$ is symmetric with respect to $\mathcal{H o m}\left(-, \omega_{\mathbb{P} n-1}\right)[n]$, hence $\omega_{\operatorname{Sec}_{d-1}}=\mathcal{O}_{\operatorname{Sec}_{d-1}}$. This also shows that $\mathrm{Sec}_{d-1}$ is arithmetically Gorenstein.

Proposition 8.15. Let $d \leq(n+1) / 2$, then $\operatorname{Sec}_{d-1}$ is smooth outside of $\operatorname{Sec}_{d-2}$. 
Proof. Let $S^{d-1} E$ be the $(d-1)$ st symmetric product of the elliptic curve $E$. Consider the incidence variety

$$
I:=\left\{(D, x) \in S^{d-1} E \times \mathbb{P}^{n-1} \mid x \in \operatorname{span}(D)\right\}
$$

and its projections

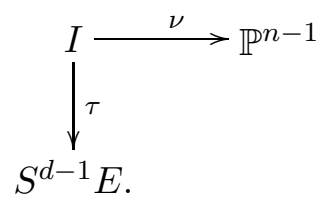

Since each divisor $D \in S^{d-1} E$ spans a $\mathbb{P}^{d-2}$ this incidence variety is a projective bundle over $S^{d-1} E$ and therefore smooth. The singular locus of $\operatorname{Sec}_{d-1}$ is therfore the union of the locus where $\nu$ is not $1: 1$ and the image of the locus in $I$ where the differential $d \nu$ is not injective.

By Lemma 13.2 the intersection of two $(d-1)$-secant $\mathbb{P}^{d-2} \mathrm{~s}$ has to be contained in a $(d-2)$-secant $\mathbb{P}^{d-3}$, so $\nu$ is $1: 1$ outside the preimage of $\operatorname{Sec}_{d-2}$. So we are left to consider the differential of $\nu$. For this we look at a divisor $D \in S^{d-1} E$ and the $d-1$-secant $\mathbb{P}^{d-2}=: \Lambda_{D}$ spanned by $D$. From now on we identify $\Lambda_{D}$ with its preimage $\nu^{-1} \Lambda_{D}$ which is the fiber of $I$ over the point $D \in S^{d-1} E$. On $\Lambda_{D}$ the tangent bundle of $I$ decomposes as

$$
\left.\mathcal{T}_{I}\right|_{\Lambda_{D}}=T_{S^{d-1} E, D} \otimes \mathcal{O}_{\Lambda_{D}} \oplus \mathcal{T}_{\Lambda_{D}}
$$

Where $T_{S^{d-1} E, D}$ is the tangent space to $S^{d-1} E$ in $D$ and $\mathcal{T}_{\Lambda_{D}}$ is the tangent bundle of the fiber $\Lambda_{D}$ over $D$.

Since $\nu$ embedds $\Lambda_{D}$ into $\mathbb{P}^{n-1}$, we have a diagram

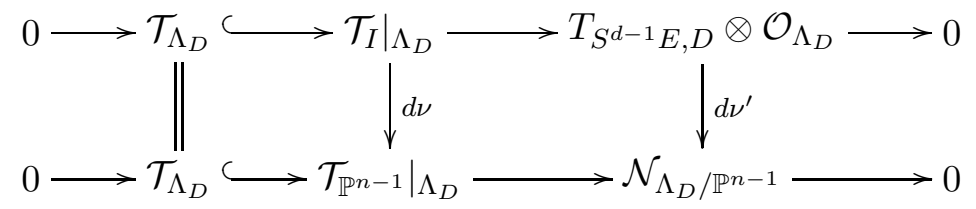

(A similar construction is used in [CS95, section 2]). For us it is now enough to show that $d \nu^{\prime}$ is injective outside of the preimage $\nu^{-1}\left(\operatorname{Sec}_{d-2} \cap \Lambda_{D}\right)$. For this we give an explict description of $d \nu^{\prime}$. First recall that $T_{S^{d-1} E, D}$ is canonically isomorphic to $H^{0}\left(\mathcal{O}_{D}(D)\right)$. (See for example [ACGH85, p. 160]). Also the linear forms on $\mathbb{P}^{n-1}=\mathbb{P}\left(H^{0}\left(\mathcal{O}_{E}(n A)\right)\right.$ that vanish on $D$ are given by the cokernel of the map

$$
H^{0}\left(\mathcal{O}_{E}(n A-D)\right) \hookrightarrow H^{0}\left(\mathcal{O}_{E}(n A)\right)
$$

induced by $D$. This cokernel is easily identified as $H^{0}\left(\mathcal{O}_{D}(n A)\right)$. Consequently we have

$$
d \nu^{\prime}: H^{0}\left(\mathcal{O}_{D}(D)\right) \otimes \mathcal{O}_{\Lambda_{D}} \rightarrow H^{0}\left(\mathcal{O}_{E}(n A-D)\right)^{*} \otimes \mathcal{O}_{\Lambda_{D}}(1)
$$

and this is induced by the restriction and multiplication of sections 


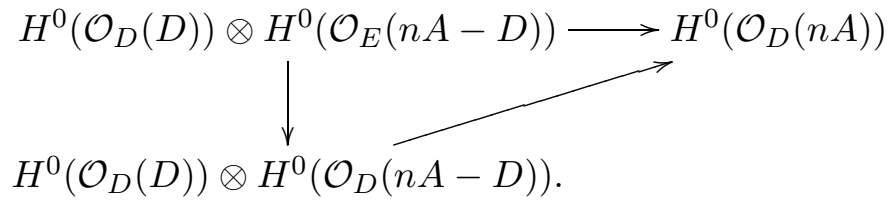

Since $2(d-1)<n$ the restriction is surjective and $d \nu^{\prime}$ factors as

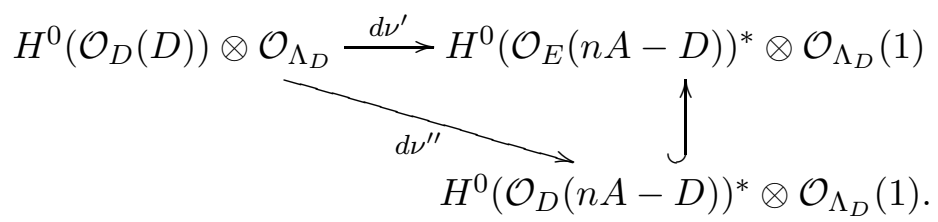

To prove injectivity of $d \nu^{\prime}$ it is enough to prove it for $d \nu^{\prime \prime}$. This map can be represented by a square matrix of linear forms. We will choose appropriate bases to do so.

For this write $D=D_{1}+\cdots+D_{m}$ with $D_{i}$ divisors supported on a single point $P_{i}$ and decompose

$$
H^{0}\left(\mathcal{O}_{D}(D)\right) \cong H^{0}\left(\mathcal{O}_{D}(n A-D)\right)=: V_{1} \oplus \cdots \oplus V_{m}
$$

where $V_{i}$ contains those sections that are supported only on $D_{i}$. The matrix $M$ representing $d \nu^{\prime \prime}$ with respect to this decomposition is made up of block matrices $M_{i j}$ representing the multiplication of sections

$$
d \nu_{i j}^{\prime \prime}: V_{i} \otimes V_{j} \rightarrow H^{0}\left(\mathcal{O}_{D}(n A)\right) .
$$

By the choice of $V_{i}$ this is zero for $i \neq j$, i.e. the block matices outside the diagonal vanish. Therfore the determinant $\operatorname{det} M$ is just the product of the determinants $\operatorname{det} M_{i i}$. Lets consider one such $M_{i i}$. $V_{i}$ has dimension $v=\operatorname{deg} D_{i}$ and a basis $\left\{1, t, \ldots, t^{v-1}\right\}$ of sections supported on $D_{i}$. The multiplication of sections $d \nu_{i i}^{\prime \prime}$ is then represented by

$$
M_{i i}=\left(\begin{array}{cccc}
1 & t & \cdots & t^{v-1} \\
t & \cdots & t^{v-1} & 0 \\
\vdots & & & \vdots \\
t^{v-1} & 0 & \cdots & 0
\end{array}\right)
$$

where the $t^{i} \in H^{0}\left(\mathcal{O}_{D}(n A)\right)$ are considered as linear forms on $\Lambda_{D}$. In particular $t^{v-1}$ is the linear form that vanishes on $P_{i}$ with multiplicity $v-1$ and on all points of $P_{j}$ with the maximal multiplicity $\operatorname{deg} D_{j}$. This cuts out the corresponding $(d-2)$-secant in $\Lambda_{D}$. So $M_{i i}$ has maximal rank outside this $(d-2)$-secant. This shows that $M$ has maximal rank outside of $\nu^{-1}\left(\operatorname{Sec}_{d-2} \cap \Lambda_{D}\right)$ as claimed.

Corollary 8.16. Let $d \leq(n+1) / 2$, then $\operatorname{Sec}_{d-1}$ is normal.

Proof. $\mathrm{Sec}_{d-1}$ is Cohen-Macaulay and smooth in codimension 1. 


\section{Poincaré linebundle}

Consider the Poincaré bundle $\mathcal{P}$ on $E \times \mathrm{Jac}_{d}$ which is uniquely determined by the normlization $\left.\mathcal{P}\right|_{\mathrm{Jac}_{d} \times\{A\}} \cong \mathcal{O}_{\mathrm{Jac}_{d}}$. Using the diagram

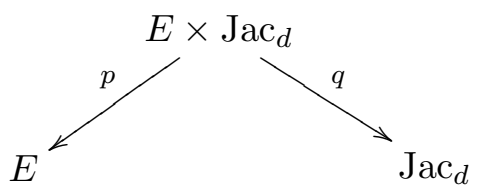

we define two vector bundles on $\mathrm{Jac}_{d}$ by setting

$$
\mathcal{G}:=q_{*}\left(p^{*} \mathcal{O}_{E}(n A) \otimes \mathcal{P}^{-1}\right) \quad \text { and } \quad \mathcal{H}:=q_{*} \mathcal{P} .
$$

The fiber of $\mathcal{G}$ over a point $\mathcal{O}_{E}(D) \in \mathrm{Jac}_{d}$ is $H^{0}(n A-D)$ and the fiber of $\mathcal{H}$ over this point is $H^{0}(D)$. Fiberwise multiplication defines a vector bundle homomorphism

$$
\mathcal{G} \otimes \mathcal{H} \rightarrow H^{0}(n A) \otimes \mathcal{O}_{E}
$$

Now projectivize $H^{0}(n A) \otimes \mathcal{O}_{E}$ to obtain a map

$$
\phi: \mathcal{G} \otimes \mathcal{O}(-1) \rightarrow \mathcal{H}^{*} \otimes \mathcal{O}
$$

on

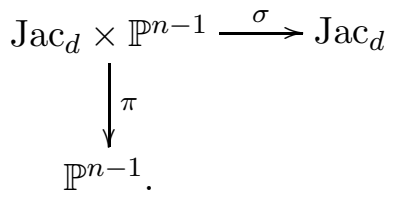

Let $X:=X_{d-1}(\phi) \in \mathrm{Jac}_{d} \times \mathbb{P}^{n-1}$ be the submaximal rank locus of $\phi$. The fiber over a point $\mathcal{O}_{E}(D) \in \mathrm{Jac}_{d}$ is the scroll $X_{|D|}$ and hence $X$ is of expected dimension. Consequently the Eagon-Northcott complex associated to $\phi$

$$
\mathcal{F}_{\phi}^{\bullet}(d): \cdots \rightarrow \mathcal{F}^{-d-1} \otimes \mathcal{O}(-d-1) \rightarrow \mathcal{F}^{-d} \otimes \mathcal{O}(-d)
$$

with terms

$$
\mathcal{F}^{-i}=\Lambda^{i} \mathcal{G} \otimes \Lambda^{d} \mathcal{H} \otimes S_{i-d} \mathcal{H}
$$

gives a resolution

$$
\mathcal{F}_{\phi}^{\bullet}(d)[-d] \rightarrow I_{X}
$$

Lemma 9.1. $\mathcal{F}_{\phi}^{\bullet}(d)$ is a family of (minimal degree) linear strands.

Proof. Over each fiber $\mathcal{F}_{\phi}^{\bullet}(d)$ restricts to the Eagon-Northcott complex that resolves $I_{X_{|D|}}$.

Proposition 9.2. Let $d<n / 2$ then we have the following identity of sheaves

$$
\pi_{*} \mathcal{O}_{X}=\mathcal{O}_{\operatorname{Sec}_{d}} \quad \text { and } \quad \pi_{*} I_{X}=I_{\text {Sec }_{d}} .
$$

In particular $\cup X=\mathrm{Sec}_{d}$ as schemes. 
Proof. Set theoretically $\mathrm{Sec}_{d}=\cup X=\pi(X)$ since every $d$-secant of $E$ cuts out a degree $d$ divisor $D$ on $E$ and hence occurs in $X_{|D|}$. Since $\operatorname{Sec}_{d}$ is normal by Corrolary 8.16 and the restriction $\left.\pi\right|_{X}: X \rightarrow \operatorname{Sec}_{d}$ is birational it follows that $\pi_{*} \mathcal{O}_{X}=\mathcal{O}_{\operatorname{Sec}_{d}}$ (cf. Har77, proof of Corollary III.11.4]). We apply $\pi_{*}$ to the exact sequence

$$
0 \rightarrow I_{X} \rightarrow \mathcal{O}_{\mathrm{Jac}_{d} \times \mathbb{P}^{n-1}} \rightarrow \mathcal{O}_{X} \rightarrow 0
$$

and obtain a long exact sequence

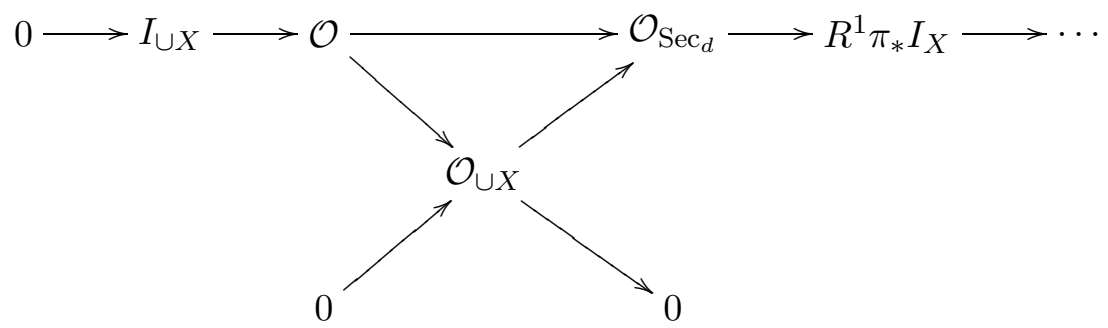

The fact that we have an injective morphism $\mathcal{O}_{\cup X} \hookrightarrow \mathcal{O}_{\operatorname{Sec}_{d}}$ shows that $\cup X$ is reduced and therefore $\cup X=\mathrm{Sec}_{d}$ as schemes.

Proposition 9.3. Let $d<n / 2$ then we have the following identity of sheaves

$$
R \mathcal{O}_{X}=\mathcal{O}_{\operatorname{Sec}_{d-1}} \quad \text { and } \quad R I_{X}=I_{\operatorname{Sec}_{d-1}} .
$$

In particular $\cap X=\mathrm{Sec}_{d-1}$ as schemes.

Proof. We shall first show that set theoretically $\cap X=\operatorname{Sec}_{d-1}$. For this note that every $(d-1)$-secant $\mathbb{P}^{d-2}$ occurs in every $X_{|D|}$, we therefore have $\operatorname{Sec}_{d-1} \subset \cap X$. Now if $n>2 d$ two $d$-secant $\mathbb{P}^{d-1}$ 's can intersect in a $\mathbb{P}^{i-1}$ if and only if this is an $i$-secant of $E$. Otherwise they would span a $\mathbb{P}^{2 d-1-i}$ containing more than $2 d-i$ points which is impossible by Lemma 13.2 Consequently we have $\operatorname{Sec}_{d-1}=\cap X$ as sets.

For an arbitrary divisor $D$ of degree $d$ we consider the diagram

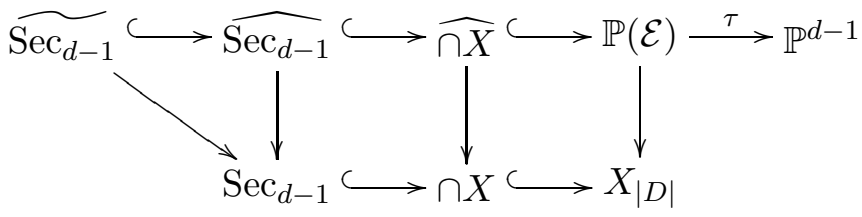

where all squares are cartesian. By Proposition 8.4 and Corrolary 8.6 the preimage $\widetilde{\operatorname{Sec}_{d-1}}$ is a divisor on $\mathbb{P}(\mathcal{E})$ of class $d H-(n-2 d) R$.

The intersection $\cap X$ is cut out by hypersurfaces $Y_{1}, \ldots Y_{m}$ of degree $d$ in $\mathbb{P}^{n-1}$. Pulling these back to $\mathbb{P}(\mathcal{E})$ gives divisors $\widehat{Y}_{i}$ of class $d H$ containing $\widehat{\cap X}$ and hence also $\widetilde{\operatorname{Sec}_{d-1}}$. This shows that

$$
\widehat{Y}_{i}=\widetilde{\operatorname{Sec}_{d-1}}+\widehat{Z}_{i}
$$

with $\widehat{Z}_{i}$ a divisor of class $(n-2 d) R$. In particular

$$
\widehat{Z}_{i}=\tau^{-1} Z_{i}
$$


where $Z_{i}$ is a hypersurface of degree $(n-2 d)$ in $\mathbb{P}^{d-1}$. Altogether we find

$$
\widehat{\cap X}=\widehat{\operatorname{Sec}_{d-1}} \cup \tau^{-1}\left(\cap Z_{i}\right)
$$

as schemes. We claim that $\cap Z_{i}=\emptyset$. Otherwise set theoretically $\widehat{\cap X}$ and with it $\widetilde{\operatorname{Sec}_{d-1}}$ contains at least one fiber of $\tau$. The image of this fiber is a $d$-secant $\mathbb{P}^{d-1}$ of $E$ contained in $\operatorname{Sec}_{d-1}$. This is a contradiction since $\operatorname{Sec}_{d-1}$ contains no $d$-secant $\mathbb{P}^{d-1}$ of $E$ by Lemma 13.2 in the appendix.

This shows $\widetilde{\operatorname{Sec}_{d-1}}=\widehat{\operatorname{Sec}_{d-1}}=\widehat{\cap X}$ and therefore also $\operatorname{Sec}_{d}=\cap X$ as schemes. Consequently

$$
R \mathcal{O}_{X}=R^{1} \pi_{*}\left(\mathcal{O}_{X} \otimes \omega_{E}\right)=\mathcal{O}_{\cap X}=\mathcal{O}_{\operatorname{Sec}_{d-1}} .
$$

As in the last proof we apply $\pi_{*}$ to the exact sequence

$$
0 \rightarrow I_{X} \rightarrow \mathcal{O}_{\mathrm{Jac}_{d} \times \mathbb{P}^{n-1}} \rightarrow \mathcal{O}_{X} \rightarrow 0
$$

to obtain the long exact sequence

$$
0 \rightarrow I_{\mathrm{Sec}_{d}} \rightarrow \mathcal{O} \stackrel{\alpha}{\rightarrow} \mathcal{O}_{\mathrm{Sec}_{d}} \rightarrow R I_{X} \rightarrow \mathcal{O} \rightarrow \mathcal{O}_{\mathrm{Sec}_{d-1}} \rightarrow 0 .
$$

Since $\alpha$ is surjective by the Proposition 9.2 above this gives $R I_{X}=I_{\operatorname{Sec}_{d-1}}$.

For future use we calculate the degree of the various vector bundles above.

Lemma 9.4. Let $\mathcal{G}$ and $\mathcal{H}$ be as above. Then $\operatorname{deg} \mathcal{G}=\operatorname{deg} \mathcal{H}=-1$.

Proof. We first have to give an explicit description of the Poincaré line bundle $\mathcal{P}$. We denote by $B \in \mathrm{Jac}_{d}$ the point corresponding to the line bundle $\mathcal{O}_{E}(d A)$. By $\Delta$ we denote the following divisor on $E \times \mathrm{Jac}_{d}$

$$
\Delta:=\left\{(P, \mathcal{L}) \mid \mathcal{L}=\mathcal{O}_{E}((d-1) A+P)\right\} .
$$

One immediately checks that

$$
\mathcal{P}=\mathcal{O}_{E \times \operatorname{Jac}_{d}}\left((d-1) p^{*} A+\Delta-q^{*} B\right) .
$$

Since $\mathrm{Jac}_{d}$ is an elliptic curve Riemann Roch gives $\chi(\mathcal{H})=\operatorname{deg} \mathcal{H}$. By the Leray spectral sequence $\chi(\mathcal{H})=\chi(\mathcal{P})$ since $R^{1} q_{*} \mathcal{P}=0$. Using Riemann Roch on the surface $E \times \mathrm{Jac}_{d}$ we obtain

$$
\chi(\mathcal{P})=\frac{1}{2}\left((d-1) p^{*} A+\Delta-q^{*} B\right)^{2}=-1 .
$$

The same reasoning works for

$$
\mathcal{G}=q_{*}\left(p^{*} \mathcal{O}_{E}(n A) \otimes \mathcal{P}^{-1}\right)=\mathcal{O}_{E \times \operatorname{Jac}_{d}}\left((n-d+1) p^{*} A-\Delta+q^{*} B\right)
$$

and an analogue calculation gives

$$
\operatorname{deg} \mathcal{G}=\chi(\mathcal{G})=-1 .
$$

Recall the following identities of exponential Chern characters on a smooth curve. 
Lemma 9.5. Let $\mathcal{F}$ be a rank $r$ bundle on a curve with exponential Chern character

$$
\operatorname{ch}(\mathcal{F})=r+c_{1}(\mathcal{F})
$$

Then

$$
\begin{aligned}
\operatorname{ch}\left(\Lambda^{k} \mathcal{F}\right) & =\left(\begin{array}{l}
r \\
k
\end{array}\right)+\left(\begin{array}{l}
r-1 \\
k-1
\end{array}\right) c_{1}(\mathcal{F}) \\
\operatorname{ch}\left(S_{k} \mathcal{F}\right) & =\left(\begin{array}{c}
r+k-1 \\
r-1
\end{array}\right)+\left(\begin{array}{c}
r+k-1 \\
r
\end{array}\right) c_{1}(\mathcal{F}) .
\end{aligned}
$$

Proof. Well known, follows from standard calculation of chern classes on curves.

Lemma 9.6. Let $\mathcal{G}, \mathcal{H}$ and

$$
\mathcal{F}^{-i}=\Lambda^{i} \mathcal{G} \otimes \Lambda^{d} \mathcal{H} \otimes S_{i-d} \mathcal{H}
$$

be as above. Then

$$
\operatorname{deg} \mathcal{F}^{-i}=-\left(\begin{array}{c}
n-d \\
i
\end{array}\right)\left(\begin{array}{l}
i \\
d
\end{array}\right) \frac{n}{n-d}
$$

Proof. Since $\mathcal{G}$ and $\mathcal{H}$ have ranks $(n-d)$ and $d$ respectively, the formulae of Lemma 9.5 give

$$
\begin{aligned}
\operatorname{ch}\left(\Lambda^{i} \mathcal{G}\right) & =\left(\begin{array}{c}
n-d \\
i
\end{array}\right)+\left(\begin{array}{c}
n-d-1 \\
i-1
\end{array}\right) c_{1}(\mathcal{G}) \\
\operatorname{ch}\left(\Lambda^{d} \mathcal{H}\right) & =1+c_{1}(\mathcal{H}) \\
\operatorname{ch}\left(S_{i-d} \mathcal{H}\right) & =\left(\begin{array}{c}
i-1 \\
d-1
\end{array}\right)+\left(\begin{array}{c}
i-1 \\
d
\end{array}\right) c_{1}(\mathcal{H}) .
\end{aligned}
$$

By the multiplicativity of the exponential Chern character with respect to tensor products, we obtain

$$
\begin{aligned}
\operatorname{ch}\left(\Lambda^{d} \mathcal{H} \otimes S_{i-d} \mathcal{H}\right) & =\left(\begin{array}{c}
i-1 \\
d-1
\end{array}\right)+\left(\begin{array}{c}
i-1 \\
d
\end{array}\right) c_{1}(\mathcal{H})+\left(\begin{array}{c}
i-1 \\
d-1
\end{array}\right) c_{1}(\mathcal{H}) \\
& =\left(\begin{array}{c}
i-1 \\
d-1
\end{array}\right)+\left(\begin{array}{c}
i \\
d
\end{array}\right) c_{1}(\mathcal{H})
\end{aligned}
$$

and

$$
\begin{aligned}
\operatorname{ch}\left(\mathcal{F}^{-i}\right) & =\operatorname{ch}\left(\Lambda^{i} \mathcal{G} \otimes \Lambda^{d} \mathcal{H} \otimes S_{i-d} \mathcal{H}\right) \\
& =\left(\begin{array}{c}
n-d \\
i
\end{array}\right)\left(\begin{array}{c}
i-1 \\
d-1
\end{array}\right)+\left(\begin{array}{c}
n-d \\
i
\end{array}\right)\left(\begin{array}{l}
i \\
d
\end{array}\right) c_{1}(\mathcal{H})+\left(\begin{array}{c}
n-d-1 \\
i-1
\end{array}\right)\left(\begin{array}{c}
i-1 \\
d-1
\end{array}\right) c_{1}(\mathcal{G}) \\
& =\left(\begin{array}{c}
n-d \\
i
\end{array}\right)\left(\begin{array}{c}
i-1 \\
d-1
\end{array}\right)+\left(\begin{array}{c}
n-d \\
i
\end{array}\right)\left(\begin{array}{l}
i \\
d
\end{array}\right)\left(c_{1}(\mathcal{H})+\frac{i}{n-d} \frac{d}{i} c_{1}(\mathcal{G})\right) \\
& =\left(\begin{array}{c}
n-d \\
i
\end{array}\right)\left(\begin{array}{c}
i-1 \\
d-1
\end{array}\right)+\left(\begin{array}{c}
n-d \\
i
\end{array}\right)\left(\begin{array}{l}
i \\
d
\end{array}\right)\left(c_{1}(\mathcal{H})+\frac{d}{n-d} c_{1}(\mathcal{G})\right)
\end{aligned}
$$

Since $\operatorname{deg}(\mathcal{H})=\operatorname{deg}(\mathcal{G})=-1$ this proves the lemma. 


\section{THE GEOMETRIC SYZYGY CONJECTURE FOR $\mathrm{Sec}_{d-1}$}

We have so far constructed the minimal free resolution of $\operatorname{Sec}_{d-1}:=\operatorname{Sec}_{d-1}$ as a mapping-cone of the minimal free resolution of $X_{|D|}$ and its dual. This exhibits roughly half of the syzygies of $\mathrm{Sec}_{d-1}$ as geometric, i.e. as coming from a scroll $X_{|D|}$ containing $\operatorname{Sec}_{d-1}$. Now this construction can be repeated for all line bundles in $\mathrm{Jac}_{d}$ and we obtain different geometric syzygies each time. In this section we will show that the geometric syzygies obtained by this procedure will generate the whole minimal free resolution of $\mathrm{Sec}_{d-1}$.

In the last section we construced a family of linear strands

$$
\mathcal{F}_{\phi}^{\bullet}(d)[-d] \rightarrow I_{X}
$$

with $\cap X=\operatorname{Sec}_{d-1}$ and $\cup X=\operatorname{Sec}_{d}$. By the procedure of Section [7 we will obtain a complex $R \mathcal{F}^{\bullet}$ that maps to the linear strand of $\cap X=\operatorname{Sec}_{d-1}$ and whose image is a part of the linear strand that is generated by geometric syzygies. We will prove that this map is surjective. The main ingredient of this proof is the fact that $\pi_{*} \mathcal{F}^{\bullet}$ vanishes since $\cup X=\operatorname{Sec}_{d}$ does not lie on any hypersurface of degree $d$.

We start by repeating our construction of the minimal free resolution of $\operatorname{Sec}_{d-1}$ for all $X_{|D|}$ at once. The relative version of Corollary 8.9 is

Proposition 10.1. If $\omega_{X}$ is the dualizing sheaf of $X$ then

$$
I_{\pi^{-1}\left(\operatorname{Sec}_{d-1}\right) / X} \cong \omega_{X}
$$

Proof. Consider the natural map

$$
\mathcal{G} \otimes \mathcal{H} \rightarrow H^{0}(n A) \otimes \mathcal{O}_{E}
$$

obtained by fiberwise multiplication of sections. Projectivizing $\mathcal{G}$ gives a short exact sequence

$$
0 \rightarrow \mathcal{H} \otimes \mathcal{O}_{\mathbb{P}(\mathcal{G})}(-1) \rightarrow H^{0}(n A) \otimes \mathcal{O}_{\mathbb{P}(\mathcal{G})} \rightarrow \mathcal{E}_{\text {rel }} \rightarrow 0
$$

The cokernel $\mathcal{E}_{\text {rel }}$ is a relative version of the bundle $\mathcal{E}$ considered in Section 8 , Notice that $\mathbb{P}(\mathcal{G})$ is just the symmetric product $S^{d-1} E$. The projectivization $\mathbb{P}\left(\mathcal{E}_{\text {rel }}\right)$ has appeared as the incidence varitey $I$ in the proof of 8.15

We have a diagram

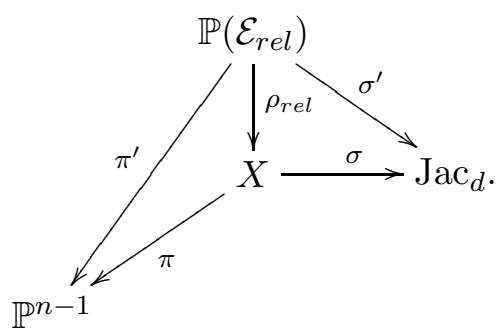

Recall that

$$
\operatorname{Sec}_{d-1} \times \operatorname{Jac}_{d}=\pi^{-1}\left(\operatorname{Sec}_{d-1}\right) .
$$

We set

$$
\operatorname{Sec}_{d-1} \times \operatorname{Jac}_{d}:=\left(\pi^{\prime}\right)^{-1}\left(\operatorname{Sec}_{d-1}\right) .
$$


Notice that over a point $|D| \in \mathrm{Jac}_{d}$ this is just the preimage

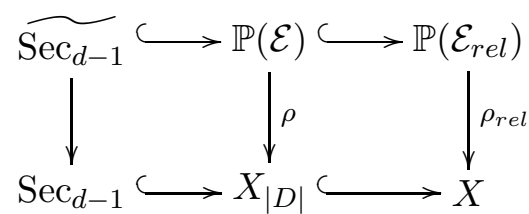

constructed in Section 8

It follows from Corollary 8.6 that

$$
\mathcal{O}_{\mathbb{P}\left(\mathcal{E}_{r e l}\right)}\left(-\operatorname{Sec}_{d-1} \times \mathrm{Jac}_{d}\right) \cong \omega_{\mathbb{P}\left(\mathcal{E}_{r e l}\right)} \otimes\left(\sigma^{\prime}\right)^{*} L
$$

for some linebundle $L$ on $\mathrm{Jac}_{d}$. Adjunction gives

$$
\left.\omega_{\operatorname{Sec}_{d-1} \times \operatorname{Jac}_{d}} \cong\left(\sigma^{\prime}\right)^{*} L\right|_{\operatorname{Sec}_{d-1} \times \operatorname{Jac}_{d}} \text {. }
$$

Pushing this forward with $\rho_{\text {rel }}$ we obtain

$$
\left.\omega_{\mathrm{Sec}_{d-1} \times \mathrm{Jac}_{d}} \cong \sigma^{*} L\right|_{\operatorname{Sec}_{d-1} \times \mathrm{Jac}_{d}} .
$$

since $\mathrm{Sec}_{d-1} \times \mathrm{Jac}_{d}$ is normal by Corollary 8.16 Now $\omega_{\mathrm{Sec}_{d-1} \times \mathrm{Jac}_{d}}$ is trivial by Corollary 8.14 and pushing down via $\sigma$ gives

$$
\mathcal{O}_{\mathrm{Jac}_{d}} \cong L \text {. }
$$

The same argument as in Lemma 8.9 then proves our claim.

Proposition 10.2. There is a short exact sequence

$$
0 \rightarrow \mathcal{O}_{\mathrm{Jac}_{d} \times \mathbb{P}^{n-1}}(-n) \rightarrow\left(\mathcal{F}_{\phi}^{\bullet}(d)\right)^{*}[-d] \rightarrow \omega_{X} \rightarrow 0
$$

with $\left(\mathcal{F}_{\phi}^{\bullet}(d)\right)^{*}=\mathcal{H o m}\left(\mathcal{F}_{\phi}^{\bullet}(d), \omega_{\operatorname{Jac}_{d} \times \mathbb{P}^{n-1}}\right)[n]$.

Proof. Applying $\mathcal{H o m}\left(-, \omega_{\mathbb{P}^{n-1} \times \mathrm{Jac}_{d}}\right)$ to the exact sequence

$$
0 \rightarrow \mathcal{F}^{\bullet}[d] \rightarrow \mathcal{O}_{\mathrm{Jac}_{d} \times \mathbb{P}^{n-1}} \rightarrow \mathcal{O}_{X}
$$

yields the desired result.

Corollary 10.3. Let $d \leq i \leq n-d$ then

$$
\left(\mathcal{F}^{-n+i}\right)^{*}=\sigma_{*}\left(\omega_{X} \otimes \pi^{*} \Omega^{i-d}(i)\right) .
$$

Proof. Since

$$
0 \rightarrow \mathcal{O}(-n) \rightarrow\left(\mathcal{F}_{\phi}^{\bullet}(d)\right)^{*}[-d] \rightarrow \omega_{X} \rightarrow 0
$$

is an exact resolution of $\omega_{X}$ and

$$
R^{q} \sigma_{*}\left(\pi^{*} \mathcal{O}(-n) \otimes \pi^{*} \Omega^{i-d}(i)\right)=H^{q}\left(\Omega^{i-d}(i-n)\right) \otimes \mathcal{O}_{\mathrm{Jac}_{d}}=0
$$

in the range $d \leq i \leq n-d$, the same spectral sequence argument as in 5.2 applies.

Each choice of $|D| \in \mathrm{Jac}_{d}$ exhibits the minimal free resolution of $\mathrm{Sec}_{d-1}$ as a mapping cone by Theorem 8.10. The information how these mapping cones fit together is encoded in the short exact sequences of the following proposition. 
Proposition 10.4. Let $d \leq i \leq n-d$. Then there are short exact sequences

$$
0 \rightarrow \mathcal{F}^{-i} \rightarrow F_{\mathrm{Sec}_{d-1}}^{-i} \otimes \mathcal{O}_{\mathrm{Jac}_{d}} \rightarrow\left(\mathcal{F}^{-n+i}\right)^{*} \rightarrow 0
$$

on $\mathrm{Jac}_{d}$

Proof. Consider the short exact sequence

$$
0 \rightarrow I_{X / \mathrm{Jac}_{d} \times \mathbb{P}^{n-1}} \rightarrow I_{\mathrm{Jac}_{d}} \times \mathrm{Sec}_{d-1} / \mathrm{Jac}_{d} \times \mathbb{P}^{n-1} \rightarrow I_{\mathrm{Jac}_{d}} \times \mathrm{Sec}_{d-1} / X \rightarrow 0 .
$$

We tensor this sequence with $\pi^{*} \Omega^{i-d}(i)$ and apply $\sigma_{*}$. By Theorem 8.10 we know that the postulation of $\operatorname{Sec}_{d-1}$ is $d$. Lemma 5.2 then tells us that

$$
\sigma_{*}\left(I_{X} \otimes \pi^{*} \Omega^{i-d}(i)\right)=\mathcal{F}^{-i} \quad \text { and } \quad R^{1} \sigma_{*} I_{X} \otimes \pi^{*} \Omega^{i-d}(i)=0 .
$$

Lemma 3.6 gives us

$$
\sigma_{*}\left(I_{\mathrm{Jac}_{d} \times \operatorname{Sec}_{d-1} / \mathrm{Jac}_{d} \times \mathbb{P}^{n-1}} \otimes \pi^{*} \Omega^{i-d}(i)\right)=F_{\mathrm{Sec}_{d-1}}^{-i} \otimes \mathcal{O}_{\mathrm{Jac}_{d}} .
$$

By Proposition 10.1

$$
I_{\mathrm{Jac}_{d} \times \operatorname{Sec}_{d-1} / X} \cong \omega_{X}
$$

and now Corollary 10.3 shows

$$
\sigma_{*}\left(\omega_{X} \otimes \pi^{*} \Omega^{i-d}(i)\right)=\left(\mathcal{F}^{-n+i}\right)^{*}
$$

This proves the result.

We shall also need the following.

Proposition 10.5. Let $d \leq i \leq n-d$, then $H^{0}\left(\mathrm{Jac}_{d}, \mathcal{F}^{-i}\right)=0$.

Proof. By Theorem 8.10 we know, that the postulation of $\operatorname{Sec}_{d}$ is $d+1$. Therefore by Proposition 6.1 we have

$$
\pi_{*} \mathcal{F}_{\phi}^{\bullet}=0,
$$

in particular

$$
\left(\pi_{*} \mathcal{F}_{\phi}^{\bullet}\right)_{i}=H^{0}\left(\mathcal{F}^{-i}\right) \otimes \mathcal{O}(-i-d)=0
$$

Theorem 10.6. The linear syzygies of $\mathrm{Sec}_{d-1}$ are spanned by scrollar syzygies.

Proof. We recall the map $\psi: R \mathcal{F}^{\bullet}(d) \rightarrow F_{\text {Sec }_{d-1}}^{\bullet}$ from Proposition 7.3 whose image is the part of the minimal free resolution of $\operatorname{Sec}_{d-1}$ generated by scrollar syzygies. We have to show that $\psi$ is surjective. For this it is enough to check that in each step the natural map

$$
\psi_{-i}: H^{1}\left(\mathcal{F}^{-i}\right) \rightarrow F_{\operatorname{Sec}_{d-1}}^{-i}
$$

is surjective. For this consider the short exact sequence

$$
0 \rightarrow \mathcal{F}^{-i} \rightarrow F_{\mathrm{Sec}_{d-1}}^{-i} \otimes \mathcal{O}_{\mathrm{Jac}_{d}} \rightarrow\left(\mathcal{F}^{-n+i}\right)^{*} \rightarrow 0 .
$$

The associated long exact sequence shows that the claim is true, if $H^{1}\left(\left(\mathcal{F}^{-n+i}\right)^{*}\right)=0$. But this follows from Proposition 10.5 since

$$
H^{1}\left(\left(\mathcal{F}^{-n+i}\right)^{*}\right)=H^{0}\left(\mathcal{F}^{-n+i}\right)
$$

by Serre duality on the elliptic curve $\operatorname{Jac}_{d}(E)$. 
We now ask wether the natural map $\psi_{-i}: H^{1}\left(\mathcal{F}^{-i}\right) \rightarrow F_{\operatorname{Sec}_{d-1}}^{-i}$ is an isomorphism. Since we already know the dimension of $F_{\operatorname{Sec}_{d-1}}^{-i}$ by Theorem 8.10, we only need to calculate $H^{1}\left(\mathcal{F}^{-i}\right)=-\operatorname{deg} \mathcal{F}^{-i}$ since $H^{0}\left(\mathcal{F}^{-i}\right)$ vanishes and $E$ is elliptic.

Corollary 10.7. $\psi: H^{1}\left(\mathcal{F}^{-i}\right) \rightarrow F_{\operatorname{Sec}_{d}}^{-i}$ is an isomorphism for $i=d$ and $i=n-d$.

Proof. Since $\psi$ is surjective, we only have to compare the dimensions of the vector spaces. By Lemma 9.6 we obtain for $i=d$

$$
H^{1}\left(\mathcal{F}^{-d}\right)=-\operatorname{deg} \mathcal{F}^{-d}=\left(\begin{array}{c}
n-d \\
d
\end{array}\right)\left(\begin{array}{l}
d \\
d
\end{array}\right) \frac{n}{n-d}
$$

since $H^{0}\left(\mathcal{F}^{-d}\right)=0$. On the other hand Corollary 8.13 yields

$$
\begin{aligned}
\operatorname{dim} F_{\operatorname{Sec}_{d-1}}^{-d} & =\left(\begin{array}{c}
n-d \\
d
\end{array}\right)\left(\begin{array}{l}
d-1 \\
d-d
\end{array}\right)+\left(\begin{array}{l}
n-d \\
n-d
\end{array}\right)\left(\begin{array}{l}
n-d-1 \\
n-d-d
\end{array}\right) \\
& =\left(\begin{array}{c}
n-d \\
d
\end{array}\right) \frac{n}{n-d}
\end{aligned}
$$

as claimed. For $i=n-d$ we obtain the same numbers.

Remark 10.8. For other steps in the resolution $\psi$ is never an isomorphism. For example if $n=6$ the the Betti diagram of $R \mathcal{F}^{\bullet}$ and $E$ are respectively

$$
\begin{array}{lllllllllllll}
- & 9 & 18 & 9 & - & - & - & - & - & 1 \\
- & 9 & 16 & 9 & - \\
1 & - & - & - & -
\end{array}
$$

The middle term of $R \mathcal{F}^{\bullet}$ is to large. In the next section we will explain this difference.

\section{THE KERNEL OF $\psi$}

As we have seen, the linear complex $R \mathcal{F}_{\phi}^{\bullet}$ is not the linear strand of $\operatorname{Sec}_{d-1}$. In this section we will describe the difference. As it turns out, this difference is the linear strand of $\mathrm{Sec}_{d}$.

Proposition 11.9. There exist an exact sequence

$$
0 \rightarrow F_{\mathrm{Sec}_{d}}^{\bullet}(d+1) \rightarrow R \mathcal{F}_{\phi}^{\bullet} \stackrel{\psi}{\rightarrow} F_{\operatorname{Sec}_{d-1}}^{\bullet}(d) \rightarrow 0
$$

Proof. Let ker ${ }^{\bullet}$ be the kernel of the surjective morphism

$$
\psi: R \mathcal{F}_{\phi}^{\bullet}(d) \rightarrow F_{\operatorname{Sec}_{d-1}}^{\bullet}(d) .
$$

Since $\psi_{-d}$ and $\psi_{-n+d}$ are isomorphisms by Corollary 10.7 we have $\mathrm{ker}^{-d}=$ $\mathrm{ker}^{-n+d}=0$, and a commutative diagram 


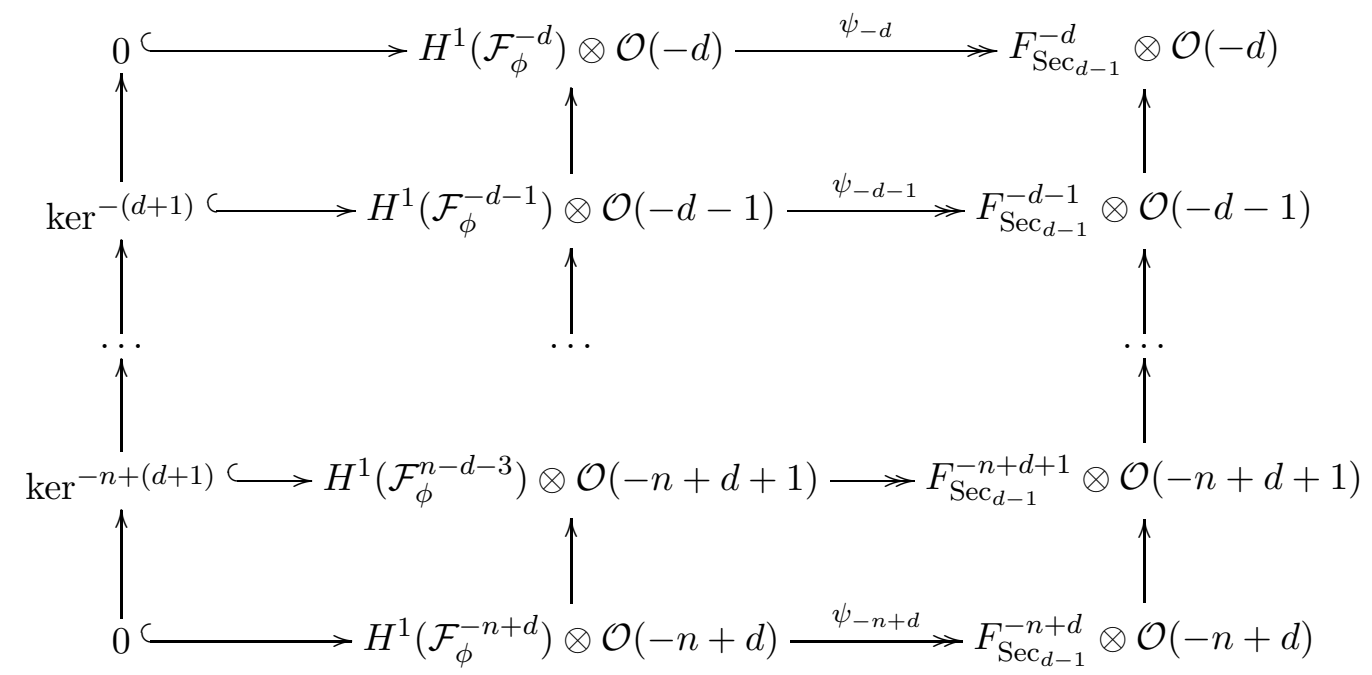

To show that ker is the degree $d+1$ linear strand of $\operatorname{Sec}_{d}$, we calculate the homology of the vertical complexes above.

For the middle column $R \mathcal{F}_{\phi}^{\bullet}(d)$ we use the hypercohomology with respect to $\pi$. Since $\mathcal{F}_{\phi}^{\bullet}(d)$ has cohomology $I_{X}$ concentrated in step $-d$, the second hypercohomology spectral sequence

$$
{ }^{I I} E_{2}^{p q}=R^{p} \pi_{*}\left(H^{q}\left(\mathcal{F}_{\phi}^{\bullet}(d)\right)\right) \Rightarrow \mathbb{H}_{\pi}^{p+q}\left(\mathcal{F}_{\phi}^{\bullet}(d)\right)
$$

yields

$$
\mathbb{H}_{\pi}^{p+q}\left(\mathcal{F}_{\phi}^{\bullet}(d)\right)=\left\{\begin{array}{cc}
\pi_{*} I_{X}=I_{\operatorname{Sec}_{d}} & \text { if } p+q=-d \\
R^{1} \pi_{*} I_{X}=I_{\operatorname{Sec}_{d-1}} & \text { if } p+q=-d+1 \\
0 & \text { otherwise. }
\end{array}\right.
$$

Consider now the first hypercohomology spectral sequence

$$
{ }^{I} E_{2}^{p q}=H^{p}\left(R^{q} \pi\left(\mathcal{F}_{\phi}^{\bullet}(d)\right)\right) \Rightarrow \mathbb{H}_{\pi}^{p+q}\left(\mathcal{F}_{\phi}^{\bullet}(d)\right) .
$$

Since

$R^{q} \pi_{*}\left(\mathcal{F}^{-i} \otimes \mathcal{O}(-i)\right)=H^{q}\left(\mathcal{F}^{-i}\right) \otimes \mathcal{O}(-i)=\left\{\begin{array}{cc}H^{1}\left(\mathcal{F}^{-i}\right) \otimes \mathcal{O}(-i) & \text { if } q=1 \\ 0 & \text { otherwise }\end{array}\right.$

this spectral sequence degenerated in level 2 and we obtain

$$
\mathbb{H}_{\pi}^{p+1}\left(\mathcal{F}_{\phi}^{\bullet}(d)\right)=H^{p}\left(R^{1} \pi_{*} \mathcal{F}_{\phi}^{\bullet}(d)\right) .
$$

Comparing this with the result from the second hypercohomology sequence shows that $R \mathcal{F}_{\phi}^{\bullet}(d)$ has cohomology $I_{\mathrm{Sec}_{d}}$ and $I_{\mathrm{Sec}_{d-1}}$ concentrated in steps $-d-1$ and $-d$.

For the right column we notice, that the minimal free resolution of $I_{\mathrm{Sec}_{d-1}}$ is

$$
0 \rightarrow \mathcal{O}(-n) \rightarrow F_{\operatorname{Sec}_{d-1} E}^{\bullet}(d) \rightarrow I_{\operatorname{Sec}_{d-1}} \rightarrow 0
$$

and therefore $F_{\operatorname{Sec}_{d-1}}^{\bullet}(d)$ has cohomology $I_{\operatorname{Sec}_{d-1}}$ in step $-d$ and $\mathcal{O}(-n)$ in step $-n+d$.

All together we obtain the long exact cohomology sequence 


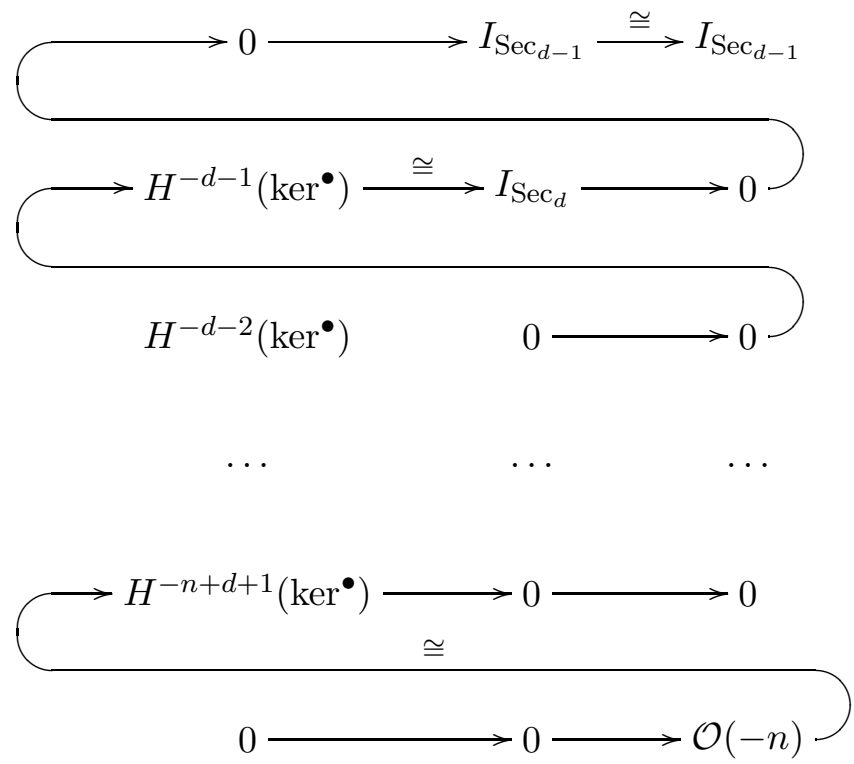

This shows that $\mathcal{O}(-n) \rightarrow \operatorname{ker}^{\bullet}[d+1] \rightarrow I_{\mathrm{Sec}_{d}}$ is exact and therefore

$$
F_{\operatorname{Sec}_{d}}^{\bullet}(d+1)=\operatorname{ker}^{\bullet}
$$

Example 11.10. For $n=6$ we can write this suggestively as

$$
\begin{aligned}
& -\quad-\begin{array}{lllllll}
- & - & - & - & - & 1 & -
\end{array}
\end{aligned}
$$

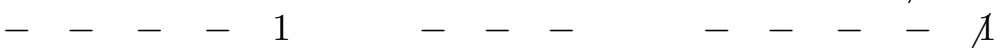

$$
\begin{aligned}
& -9169-\quad+\quad-2-\quad=\quad-\quad 9189-
\end{aligned}
$$

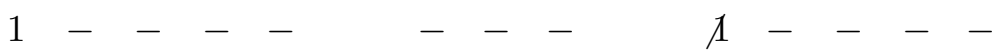

$$
\begin{aligned}
& 1--\quad-1--
\end{aligned}
$$

(In a Betti diagram all entries on a diagonal have the same twist, therefore one can sometimes "cancel diagonally".)

Example 11.11. For $n=7$ we write similarly

$$
\begin{array}{ccccccccccccccccccc}
- & - & - & - & - & 1 & & - & - & - & 1 & & - & - & - & - & 1 & - \\
- & 14 & 35 & 35 & 14 & - & + & - & 7 & 7 & - & = & - & 14 & 42 & 42 & 14 & - \\
1 & - & - & - & - & - & & - & - & - & - & & 1 & - & - & - & - & - \\
1 & - & - & - & & - & 1 & - & - & - & -
\end{array}
$$

12. The geometric Syzygy Conjecture for Bielliptic Canonical CURVES

As an application of our study of elliptic normal curves, we deduce the geometric syzygy conjecture for bielliptic canonical curves. This will follow directly from the classic fact, that a bielliptic canonical curve $C$ is the transversal intersection of a quadric $Q$ in $\mathbb{P}^{g-1}$ with a cone $\tilde{E}$ over an elliptic normal curve $E$ of degree $g-1$. From this we obtain the minimal free resolution of $\mathcal{O}_{C}$ as the total complex of

$$
\left[F_{Q}^{\bullet}[1] \rightarrow \mathcal{O}_{\mathbb{P}^{g-1}}\right] \otimes\left[F_{\tilde{E}}^{\bullet}[1] \rightarrow \mathcal{O}_{\mathbb{P}^{g-1}}\right]
$$


where $F_{Q}^{\bullet}$ and $F_{\tilde{E}}^{\bullet}$ are the minimal free resolutions of $I_{Q}$ and $I_{\tilde{E}}$. In particular, all higher syzygies of $C$ come from syzygies of $\tilde{E}$, which in turn come from geometric syzygies.

Let us start by recalling the classical facts.

Proposition 12.1. Let $C \subset \mathbb{P}^{g-1}$ be a bielliptic canonical curve of genus $g$ and Clifford index $c \geq 2$. Let $\iota: C \stackrel{2: 1}{\longrightarrow} E$ be the elliptic involution. Then

$$
\tilde{E}=\bigcup_{e \in E} \operatorname{span}\left(\iota^{-1}(e)\right)
$$

is an elliptic cone with vertex $p \notin C$. The projection of $\tilde{E}$ from $p$ is an elliptic normal curve $E \subset \mathbb{P}^{g-2}$. Moreover there exists a (not uniquely defined) quadric $Q \subset \mathbb{P}^{g-1}$ such that

$$
C=\tilde{E} \cap Q
$$

and this intersection is transversal.

Proof. The fact that all lines $\operatorname{span}\left(\iota^{-1} e\right)$ contain a common point $p \notin C$ is ACGH85, p. 269, Exercise E-1]. Projecting from this point gives an elliptic curve $E \subset \mathbb{P}^{g-2}$. The degree of $E$ is $g-1$ since the projection also induces the 2: 1 map from the degree $2 g-2$ curve $C$ to $E$. Consequently $E$ is an elliptic normal curve, and $I_{\tilde{E}}$ is the pullback of $I_{E}$ via the projection from $p$. From Theorem 8.10 respectively Corollary 8.13 applied to $E=\mathrm{Sec}_{1}$ we know that $I_{E}$ is generated by

$$
\left(\begin{array}{c}
g-3 \\
2
\end{array}\right) \frac{g-1}{g-3}=\left(\begin{array}{c}
g-2 \\
2
\end{array}\right)-1
$$

quadrics. On the other hand by Petri's Theorem [ACGH85, p.131] the ideal of every non-trigonal canonical curve without a $g_{5}^{2}$ is generated by $\left(\begin{array}{c}g-2 \\ 2\end{array}\right)$ quadrics. Consequently there exist a quadric $Q$ that contains $C$ but not $\tilde{E}$. The intersection $\tilde{E} \cap Q$ is then transversal and yields $C$.

Corollary 12.2. The minimal free resolution of $C \subset \mathbb{P}^{g-1}$ is the total complex of

$$
\left[\mathcal{O}_{\mathbb{P}^{g-1}}(-2) \stackrel{Q}{\rightarrow} \mathcal{O}_{\mathbb{P}^{g-1}}\right] \otimes\left[\mathcal{O}_{\mathbb{P}^{g-1}}(-g-1) \rightarrow \iota^{*} F_{E}^{\bullet}(2)[-1] \rightarrow \mathcal{O}_{\mathbb{P}^{g-1}}\right]
$$

where $F_{E}^{\bullet}(2)$ is the linear strand of $E \subset \mathbb{P}^{g-2}$.

Proof. Since $\tilde{E}$ is a cone over $E$, the pullback of the minimal free resolution of $E$

$$
\mathcal{O}_{\mathbb{P}^{g-2}}(-g+1) \rightarrow F_{E}^{\bullet}(2)[-1] \rightarrow \mathcal{O}_{\mathbb{P}^{g-2}}
$$

via the projection from the vertex $p$ gives the minimal free resolution of $\tilde{E}$. Now the intersection $C=Q \cap \tilde{E}$ is transversal, so the total complex above gives a free resolution of $C$. Since $C \subset \tilde{E}$ and both have linear the same postulation, $F_{\tilde{E}}^{\bullet}(2)$ must be a subcomplex of the minimal free resolution of $C$. Therefore no canceling in the above total complex can occur, i.e. the total complex is a minimal free resolution of $\mathcal{O}_{C}$. 
Example 12.3. For $g=7$ we obtain the following Betti diagrams

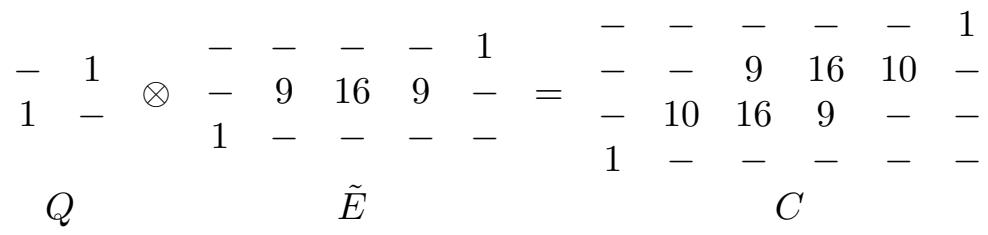

Corollary 12.4. Let $C \in \mathbb{P}^{g-1}$ be a bielliptic canonical curve of Clifford index $c \geq 2$. Then the geometric syzygy conjecture is true for every step of the minimal free resolution of $C$.

Proof. By a theorem of Green Gre84b the rank 4 quadrics in the ideal of any canonical curve $C$ span the degree 2 part of $I_{C}$. This is the geometric syzygy conjecture in step 0 . By the preceding proposition all other terms in the linear strand $F_{C}^{\bullet}(2)$ come from the minimal free resolution of $E$ and are therefore spanned by geometric syzygies.

Remark 12.5. More precisely we have shown, that the higher linear syzygies of $C$ are generated by syzygies of scrolls constructed from the $g_{4}^{1}$ 's of $C$ that factor over a $g_{2}^{1}$ on $E$.

Remark 12.6. The geometric syzygy conjecture is known for curves of Clifford index 1, i.e. trigonal curves Eus94 and curves which are isomorphic to plane quintics. For trigonal curves it is again enough to consider scrollar syzygies, but for plane quintics the scrollar syzygies no longer suffice and one has to take other geometric syzygies into account. See for example vB00 p. 87, Bemerkung 5.5.4].

\section{APENDIX}

Lemma 13.1. The cohomology of $\Omega^{i}(i+j)$ is concentrated in one degree. More explicitly it is zero except for the following cases:

\begin{tabular}{|c|c|}
\hline twist & $\begin{array}{c}\text { non zero } \\
\text { cohomology }\end{array}$ \\
\hline$j<-n-1$ & $H^{n}$ \\
$j=-i$ & $H^{i}$ \\
$j>0$ & $H^{0}$ \\
\hline
\end{tabular}

Moreover $H^{i}\left(\Omega^{i}\right)=1$.

Proof. Use the Theorem of Bott.

Lemma 13.2. Let $E \subset \mathbb{P}^{n-1}$ be an elliptic normal curve of degree $n$. Then every (possibly non-reduced) divisor $D$ of degree $d \leq n-1$ spans a linear subspace of dimension $d-1$

Proof. Assume that this statement is false. Then given any $n-d$ points $P_{1}, \ldots, P_{n-d}$ there exists a hyperplane $H$ which contains $D$ and the points $P_{1}, \ldots, P_{n-d}$. On the other hand $D+P_{1}+\ldots P_{n-d}$ is not linearly equivalent to a hyperplane section if the $P_{i}$ are chosen generically. This gives a contradiction. 


\section{REFERENCES}

[ACGH85] E. Arbarello, M. Cornalba, P.A. Griffiths, and J. Harris. Geometry of Algebraic Curves. Grundlehren der mathematischen Wissenschaften 129. Springer, Heidelberg, 1985.

[CS95] C. Ciliberto and E. Sernesi. Singularities of the Theta Divisor and Families of Secant Spaces to a Canonical Curve. Journal of Algebra, 171:867-893, 1995.

[Ehb94] S. Ehbauer. Syzygies of points in projective space and applications. In F. Orecchia, editor, Zero-dimensional schemes. Proceedings of the international conference held in Ravelo, Italy, June 8-13, 1992, pages 145-170, Berlin, 1994. de Gruyter.

[Eis88] D. Eisenbud. Linear sections of determinantal varieties. Amer. J. Math., 110(3):541-575, 1988

[Eus94] F. Eusen. Der Ansatz von Paranjape und Ramanan bei der Vermutung von Green. Dissertation, Universität Hannover, 1994.

[Gre84a] M.L. Green. Koszul cohomology and the geometry of projective varieties. J. Differential Geometry, 19:125-171, 1984.

[Gre84b] M.L. Green. Quadrics of rank four in the ideal of a canonical curve. Inv. Math., 75:85-104, 1984.

[GS] Daniel R. Grayson and Michael E. Stillman. Macaulay 2, a software system for research in algebraic geometry. Available at http://www.math.uiuc.edu/Macaulay2

[Har77] R. Hartshorne. Algebraic Geometry. Graduate Texts in Math. 52. Springer, Heidelberg, 1977.

[ML84] M. and R. Lazarsfeld. The non-vanishing of certain Koszul cohomology groups. J. Diff. Geom., 19:168-170, 1984.

[Sch86] F.O. Schreyer. Syzygies of canonical curves and special linear series. Math. Ann., 275:105-137, 1986.

[TiB02] Montserrat Teixidor i Bigas. Green's conjecture for the generic $r$-gonal curve of genus $g \geq 3 r-7$. Duke Math. J., 111(2):195-222, 2002.

[vB00] H.-Chr. Graf v. Bothmer. Geometrische Syzygien von kanonischen Kurven. Dissertation, Universität Bayreuth, 2000.

[vB02] H.-Chr. Graf v. Bothmer. Geometric syzygies of Mukai varieties and general canonical curves with genus $\leq 8$. math.AG/0202133 2002.

[Voi02] Claire Voisin. Green's generic syzygy conjecture for curves of even genus lying on a K3 surface. J. Eur. Math. Soc. (JEMS), 4(4):363-404, 2002.

[Voi03] C. Voisin. Green's canonical syzygy conjecture for generic curves of odd genus. math.AG/0301359 2003.

[Wei94] Charles A. Weibel. An introduction to homological algebra, volume 38 of Cambridge Studies in Advanced Mathematics. Cambridge University Press, Cambridge, 1994.

Institut für Mathematik, Universität Hannover, Postfach 6009, 30060 HanNOVER

E-mail address: bothmer@math.uni-hannover.de, hulek@math.uni-hannover.de 